\title{
Discrete Hilbert boundary value problems on half lattices
}

\section{P. Cerejeiras, U. Kähler \& M. Ku}

To cite this article: P. Cerejeiras, U. Kähler \& M. Ku (2015): Discrete Hilbert boundary value problems on half lattices, Journal of Difference Equations and Applications, DOI: 10.1080/10236198.2015.1071804

To link to this article: http://dx.doi.org/10.1080/10236198.2015.1071804

\section{Published online: 02 Nov 2015.}

Submit your article to this journal $₫$

Џ Article views: 1

Q View related articles $\square$

View Crossmark data ¿ 


\title{
Discrete Hilbert boundary value problems on half lattices
}

\author{
P. Cerejeiras ${ }^{1}$, U. Kähler ${ }^{2}$ and M. Ku* \\ CIDMA, Department of Mathematics, University of Aveiro, Portugal
}

(Received 2 February 2015; accepted 5 July 2015)

\begin{abstract}
We study discrete Hilbert boundary value problems in the case of the upper half lattice. The solutions are given in terms of the discrete Cauchy transforms for the upper and lower half space while the study of their solvability is based on the discrete Hardy decomposition for the half lattice. Furthermore, the solutions are proved to converge to those of the associated continuous Hilbert boundary value problems.
\end{abstract}

Keywords: discrete Dirac operator; discrete potential theory; discrete Hilbert problem; discrete Fourier transform

MSC (2010) Classification: primary: 39A12; secondary: 31C20, 65T50

\section{Introduction}

The Hilbert problem is a classic topic in complex analysis. The question of determining aholomorphic function by its boundary values is linked to many problems in continuum mechanics, in hydrodynamics or in materials with memory. Its solvability in the framework of complex analysis were studied in the classical papers of F.D. Gakhov, I.N. Vekua, N.I. Mishkelishvili, B.V. Khvedekidze, D.A. Kveselava and others (see, e.g. $[12,17,21])$. Later on it was extended to higher dimensions by S. Bernstein and others in the framework of Clifford analysis (cf. [2,5,15]). These higher-dimensional Hilbert problems are linked not only to problems in continuum mechanics, but also to other areas like image processing, where the notion of monogenic signal corresponds to the solution of a Hilbert problem.

Recently, there is an increased interest in constructing discrete counterparts of continuous structures. Such connections were successfully employed by S. Smirnov and D. Chelkak in their study of discrete Riemann problems with respect to discrete holomorphic functions in connection with the 2D-Ising model, e.g. in [7,18]. But, although discrete complex analysis was studied since the 1940s higher-dimensional analogues of the discrete Cauchy-Riemann equations only appeared in the 80s and 90s starting with Becher and Joos (cf. [1]).

The development of the corresponding function theory, as a generalization of discrete analytic function theory into higher dimensions, also called theory of discrete monogenic function or discrete Clifford analysis, has started quite recently, see, for instance, $[3,6,8,10,11,14]$. Among others, the discrete fundamental solution to the discrete Dirac operator and discrete Cauchy formula were constructed $([3,6,13])$ with the potential for future applications of said theory being illustrated in [4,9]. Since in their last paper [6] the authors constructed the corresponding discrete Hardy space, the natural question arises as how do discrete Hilbert problems would look like. This in not just a purely theoretical

*Corresponding author. Email: kumin0844@163.com 
question, since such problems are closely linked to problems in elasticity, in particular to problems related to materials with memory. Although such problems usually are modelled as continuous problems they can be, and indeed are, initially modelled as discrete problems over a lattice. Moreover, to obtain its solution one reduces the continuous problem again to a discrete one. Furthermore, such discrete problems are also linked to discrete physical applications, like the Ising model [7,18] or problems in quantum mechanics. Motivated by these considerations, we present a first version of discrete Hilbert boundary value problems with respect to the Dirac operator in higher dimensions. We show that such problems can be studied by methods similar to the ones in the continuous case. Their solutions are given in terms of discrete Cauchy transforms for the upper and lower half space while the study of their solvability is based on the discrete Hardy decomposition for the half lattice. We end the paper with the study of the convergence of the solution of the discrete Hilbert problem to the solution of its continuous counterpart.

\section{Preliminaries}

\subsection{Notations}

For the grid $h \mathbb{Z}^{n}$, where $0<h<1$ denotes the lattice constant, or mesh size, the standard forward and backward differences $\partial_{h}^{ \pm j}$ are given by

$$
\partial_{h}^{ \pm j} f(h m)= \pm \frac{1}{h}\left[f\left(h m \pm h e_{j}\right)-f(h m)\right]= \pm \frac{1}{h}\left(T_{h}^{ \pm j}-1\right) f(h m),
$$

where $\left\{e_{j}, j=1,2, \cdots, n\right\}$ denotes an orthonormal basis, $T_{h}^{ \pm j}$ denotes the translation operators and $h m=h \sum_{j=1}^{n} m_{j} e_{j} \in h \mathbb{Z}^{n}$. Based on the forward and backward differences one obtains the star-Laplacian

$$
\Delta_{h}=\sum_{j=1}^{n} \partial_{h}^{+j} \partial_{h}^{-j}=\sum_{j=1}^{n} \partial_{h}^{-j} \partial_{h}^{+j}
$$

By splitting each basis element $e_{j}$ into two new basis elements (each corresponding to the forward and backward directions) $e_{j}^{+}$and $e_{j}^{-}$satisfying to $e_{j}=e_{j}^{+}+e_{j}^{-}$we obtain new basis elements satisfying to

$$
e_{j}^{-} e_{k}^{-}+e_{k}^{-} e_{j}^{-}=e_{j}^{+} e_{k}^{+}+e_{k}^{+} e_{j}^{+}=0, \quad e_{j}^{+} e_{k}^{-}+e_{k}^{-} e_{j}^{+}=-\delta_{j k},
$$

where $\delta_{j k}$ is the delta - Kronecker symbol. These elements generate a free algebra which is isomorphic to the complexified Clifford algebra $\mathbb{C}_{n}$ (see, e.g. [8,10,11]).

In what follows we consider functions defined on $(\varnothing \neq) \Omega \subset h \mathbb{Z}^{n}$ with values in $\mathbb{C}_{n}$. Properties like $\ell_{p}$-summability $(1 \leq p<\infty)$, etc., are defined for a $\mathbb{C}_{n}$-valued function by ascribing it to each component. The corresponding spaces of functions are denoted by $\ell_{p}\left(\Omega, \mathbb{C}_{n}\right)(1 \leq p<+\infty)$, and so on. If no ambiguity arises we shall omit the range space, e.g. $\ell_{p}\left(h \mathbb{Z}^{n}, \mathbb{C}_{n}\right)=\ell_{p}\left(h \mathbb{Z}^{n}\right)$. Otherwise, it will be explicitly stated.

For more details we refer the reader to the existent literature, e.g. $[3,8,11,14,13,4]$.

The discrete Dirac operator $D^{+-}$and its adjoint $D^{-+}$are given by

$$
D_{h}^{+-}=\sum_{j=1}^{n} e_{j}^{+} \partial_{h}^{+j}+e_{j}^{-} \partial_{h}^{-j}, \quad D_{h}^{-+}=\sum_{j=1}^{n} e_{j}^{+} \partial_{h}^{-j}+e_{j}^{-} \partial_{h}^{+j},
$$


and they factorize the star-Laplacian, i.e. $\left(D_{h}^{+-}\right)^{2}=\left(D_{h}^{-+}\right)^{2}=-\Delta_{h}$. A function $f \in \ell_{1}(\Omega)$ is said to be a (left) discrete monogenic function in $\Omega$ if $D^{+-} f=0$, in $\Omega$.

Also of importance is the discrete Fourier transform

$$
\mathcal{F}_{h}: \ell_{2}\left(h \mathbb{Z}^{n}\right) \rightarrow L_{2}\left(\left[-\frac{\pi}{h}, \frac{\pi}{h}\right]^{n}\right),
$$

given pointwisely as

$$
f(h m) \mapsto \mathcal{F}_{h} f(x)=\left\{\begin{array}{cl}
\sum_{m \in \mathbb{Z}^{n}}^{n} e^{i(h m, x)} f(h m) h^{n}, & x \in\left[-\frac{\pi}{h}, \frac{\pi}{h}\right]^{n} \\
0, & x \notin\left[-\frac{\pi}{h}, \frac{\pi}{h}\right]^{n}
\end{array}\right.
$$

with $\langle h m, x\rangle:=h \sum_{j=1}^{n} m_{j} x_{j}$. The discrete Fourier transform $\mathcal{F}_{h}$ has an inverse given by $R_{h} \mathcal{F}$, the restriction $R_{h}$ to the lattice of the (standard) continuous Fourier transform

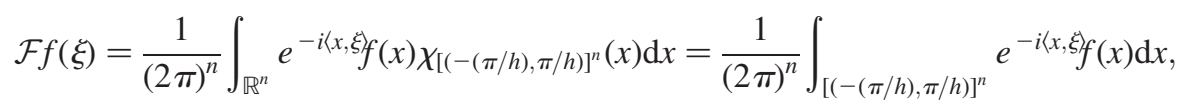

acting on functions $f$ with support in the hyper-cube $[-(\pi / h), \pi / h]^{n}$.

The fundamental solution $E_{h}^{-+}$of $D_{h}^{-+}$, that is, the discrete Cauchy kernel, is given in terms of its Fourier transform as

$$
E_{h}^{-+}=R_{h} \mathcal{F}\left(\frac{\xi^{D}}{d^{2}}\right)=\sum_{j=1}^{n} e_{j}^{+} R_{h} \mathcal{F}\left(\frac{\xi_{-j}^{D}}{d^{2}}\right)+e_{j}^{-} R_{h} \mathcal{F}\left(\frac{\xi_{+j}^{D}}{d^{2}}\right)
$$

with $\xi_{ \pm j}^{D}= \pm h^{-1}\left(e^{\mp i h \xi_{j}}-1\right)$, and where $\xi^{D}=\sum_{j=1}^{n} e_{j}^{+} \xi_{-j}^{D}+e_{j}^{-} \xi_{+j}^{D}$ and $d^{2}=$ $4 / h^{2} \sum_{j=1}^{n} \sin ^{2}\left(\xi_{j} h / 2\right)$ denote the symbol of the discrete Dirac operator $D^{-+}$and of the negative of the star-Laplacian (that is to say, $\left.\mathcal{F}_{h}\left(-\Delta_{h} f\right)=d^{2} \mathcal{F}_{h} f\right)$, respectively. The discrete Cauchy kernel has the following properties:

LEMMA 2.1. The discrete Cauchy kernel $E_{h}^{-+}$satisfies

(i) $D_{h}^{-+} E_{h}^{-+}(h m)=\delta_{h}(h m), \quad h m \in h \mathbb{Z}^{n}$,

(ii) $E_{h}^{-+} \in \ell_{p}\left(\mathbb{Z}^{n}\right), \quad p>\frac{n}{n-1}$,

where $\delta_{h}$ denotes the discrete delta of Dirac function in $h \mathbb{Z}^{n}$ defined as

$$
\delta_{h}(h m)=\left\{\begin{array}{clc}
h^{-n} & \text { if } \quad h m=0 \\
0 & \text { if } \quad h m \neq 0
\end{array}\right.
$$

The proof will be omitted, as it follows the same argument of Lemma 2.7 adapted to dimension $n$ (also, cf. [13], [19]).

\subsection{Discrete Hardy spaces}

In the section, we provide a short overview on discrete Hardy spaces. For the proofs we refer to [6]. Although in that paper the proofs are given only for the special case of $n=3$ their adaptation to the general case is straightforward. 
Let $m=\underline{m}+m_{n} \in \mathbb{Z}^{n}$. We define the upper/lower half spaces as

$$
\begin{gathered}
h \mathbb{Z}_{+}^{n}=\left\{h m \in h \mathbb{Z}^{n}: m_{n}>0\right\}, \quad h \mathbb{Z}_{+, 0}^{n}=\left\{h m \in h \mathbb{Z}^{n}: m_{n} \geq 0\right\}, \\
h \mathbb{Z}_{-}^{n}=\left\{h m \in h \mathbb{Z}^{n}: m_{n}<0\right\}, \quad h \mathbb{Z}_{+, 0}^{n}=\left\{h m \in h \mathbb{Z}^{n}: m_{n} \leq 0\right\} .
\end{gathered}
$$

Based on the Stokes' formula and our Cauchy kernel we have the following result.

THEOREM 2.2. Let $f$ be a discrete left monogenic function $\left(D_{h}^{+-} f=0\right)$. Then, the upper discrete Cauchy formula

$$
\begin{aligned}
& \sum_{\underline{\eta} \in \mathbb{Z}^{n-1}}\left[E_{h}^{-+}\left(h\left(\underline{\eta}-\underline{m},-m_{n}\right)\right) e_{n}^{+} f(h(\underline{\eta}, 1))+E_{h}^{-+}\left(h\left(\underline{\eta}-\underline{m}, 1-m_{n}\right)\right) e_{n}^{-} f(h \underline{\eta}, 0)\right] h^{n-1} \\
& \quad=\left\{\begin{array}{ccc}
0, & \text { if } & m_{n} \leq 0, \\
-f(h m), & \text { if } & m_{n}>0,
\end{array}\right.
\end{aligned}
$$

respectively, the lower discrete Cauchy formula

$$
\begin{aligned}
& \sum_{\underline{\eta} \in \mathbb{Z}^{n-1}}\left[E_{h}^{-+}\left(h\left(\underline{\eta}-\underline{m},-1-m_{n}\right)\right) e_{n}^{+} f(h \underline{\eta}, 0)+E_{h}^{-+}\left(h\left(\underline{\eta}-\underline{m},-m_{n}\right)\right) e_{n}^{-} f(h(\underline{\eta},-1))\right] h^{n-1} \\
& \quad=\left\{\begin{array}{ccc}
0, & \text { if } \quad m_{n} \geq 0, \\
f(h m), & \text { if } \quad m_{n}<0,
\end{array}\right.
\end{aligned}
$$

hold provide that the involved series converge.

Since the boundary value of a function defined on the upper half lattice consists of its values in two specific layers we shall denote the boundary data of $f \in \ell_{p}\left(h \mathbb{Z}_{+, 0}^{n}\right)$, or $\left.f\right|_{m_{n}=0^{+}}$, as the pair $\left(e_{n}^{-} f^{0}, e_{n}^{+} f^{1}\right)$, where $f^{0}(h \underline{m})=f(h(\underline{m}, 0))$ and $f^{1}(h \underline{m})=f(h(\underline{m}, 1))$. Given $\left.f\right|_{m_{n}=0^{+}}=\left(e_{n}^{-} f^{0}, e_{n}^{+} f^{1}\right)$ (a pair of functions in $\ell_{p}\left(h \mathbb{Z}^{n-1}, \mathbb{C}_{n}\right)$ ), its upper discrete Cauchy transform is given by

$$
\begin{aligned}
C^{+} & {\left[e_{n}^{-} f^{0}, e_{n}^{+} f^{1}\right](h m) } \\
& =-\sum_{\underline{\eta} \in \mathbb{Z}^{n-1}}\left[E_{h}^{-+}\left(h\left(\underline{\eta}-\underline{m},-m_{n}\right)\right) e_{n}^{+} f^{1}(h \underline{\eta})+E_{h}^{-+}\left(h\left(\underline{\eta}-\underline{m}, 1-m_{n}\right)\right) e_{n}^{-} f^{0}(h \underline{\eta})\right] h^{n-1},
\end{aligned}
$$

for all $m \in \mathbb{Z}^{n}$

In a similar way, the boundary data of $f \in \ell_{p}\left(h \mathbb{Z}_{-, 0}^{n}\right)$, or $\left.f\right|_{m_{n}=0^{-}}$, will be the pair $\left(e_{n}^{+} f^{0}, e_{n}^{-} f^{-1}\right)$, where $f^{0}(h \underline{m})=f(h(\underline{m}, 0))$ and $f^{-1}(h \underline{m})=f(h(\underline{m},-1) \quad$ Given 
$\left.f\right|_{m_{n}=0^{-}}=\left(e_{n}^{+} f^{0}, e_{n}^{-} f^{-1}\right)$, its lower discrete Cauchy transform is given by

$$
\begin{aligned}
C^{+} & {\left[e_{n}^{+} f^{0}, e_{n}^{-} f^{-1}\right](h m) } \\
& =-\sum_{\underline{\eta} \in \mathbb{Z}^{n-1}}\left[E_{h}^{-+}\left(h\left(\underline{\eta}-\underline{m},-1-m_{n}\right)\right) e_{n}^{+} f^{0}(h \underline{\eta})+E_{h}^{-+}\left(h\left(\underline{\eta}-\underline{m},-m_{n}\right)\right) e_{n}^{-} f^{-1}(h \underline{\eta})\right] h^{n-1},
\end{aligned}
$$

for all $m \in \mathbb{Z}^{n}$

This unusual concept of boundary data is motivated on the one hand by the fact that the discrete setting requires two discrete derivatives - backward and forward - each acting on two layers of $f$, and on the second hand by the fact that $e_{n}^{+}, e_{n}^{-}$are nilpotent elements which in turns implies only these parts of the boundary data functions are indeed relevant for our discrete Cauchy transforms.

For the upper and lower discrete Cauchy transforms the following properties are valid.

THEOREM 2.3. The upper and lower Cauchy transforms, (6) and (7), respectively, satisfy

(i) $C^{+}\left[e_{n}^{-} f^{0}, e_{n}^{+} f^{1}\right] \in \ell_{p}\left(h \mathbb{Z}^{n}\right), \quad\left[C^{-} e_{n}^{+} f^{0}, e_{n}^{-} f^{-1}\right] \in \ell_{p}\left(h \mathbb{Z}^{n}\right)$, for all $1 \leq p<$ $+\infty$;

(ii) $D_{h}^{+-} C^{+}\left[e_{n}^{-} f^{0}, e_{n}^{+} f^{1}\right](h m)=0$, for all $m=\left(\underline{m}, m_{n}\right) \in h \mathbb{Z}^{n}$ satisfying to $m_{n}>1$;

(iii) $D_{h}^{+-} C^{-}\left[e_{n}^{+} f^{0}, e_{n}^{-} f^{-1}\right](h m)=0$, for all $m=\left(\underline{m}, m_{n}\right) \in h \mathbb{Z}^{n}$ satisfying to $m_{n}<-1$.

Based on the Fourier symbols of the fundamental solution in the (-1)-, 0- and 1-layers the authors obtained the discrete upper and lower Hilbert transforms (cf. [6] for more details)

$$
\begin{gathered}
H_{+} f=\mathcal{F}_{h}^{-1}\left[\frac{\underline{\underline{\xi}}}{\underline{d}}\left(e_{n}^{+} \frac{h \underline{d}-\sqrt{4+h^{2} \underline{d}^{2}}}{2}+e_{n}^{-} \frac{2}{h \underline{d}-\sqrt{4+h^{2} \underline{d}^{2}}}\right)\right] \mathcal{F}_{h} f, \\
H_{-} f=-\mathcal{F}_{h}^{-1}\left[\left[\frac{\underline{\xi}^{D}}{\underline{d}}\left(e_{n}^{+} \frac{2}{h \underline{d}-\sqrt{4+h^{2} \underline{d}^{2}}}+e_{n}^{-} \frac{h \underline{d}-\sqrt{4-h^{2} \underline{d}^{2}}}{2}\right)\right] \mathcal{F}_{h} f,\right.
\end{gathered}
$$

where $\mathcal{F}_{h}$ denotes the $(n-1)$ - dimensional discrete Fourier transform and both $\underline{\xi}^{D}, \underline{d}$ denote the symbols of the $n-1$ dimensional discrete Dirac and star-Laplacian, respectively. This allows us to give the following definition of discrete Hardy spaces.

DEFINITION 2.4. We define the discrete Hardy spaces $h_{p}^{ \pm}$as the spaces of all discrete functions $f \in \ell_{p}\left(h \mathbb{Z}^{n-1}\right)$ satisfying to

$$
P^{+} f=\frac{1}{2}\left(1+H_{ \pm}\right) f=f,
$$

respectively.

These conditions can be thought of as the discrete equivalents of the continuous Plemelj-Sokhotzki formulae. However, due to the fact that the discrete boundary data consists of two layers the Hardy space decomposition is slightly different in the sense that we have to take into account discrete complementary Hardy spaces. 
DEFINITION 2.5. We define the discrete complementary Hardy spaces $\bar{h}_{p}^{ \pm}$as the spaces of all discrete functions $f \in \ell_{p}\left(h \mathbb{Z}^{n-1}\right)$ satisfying

$$
Q^{ \pm} f:=\frac{1}{2}\left(1-H_{ \pm}\right) f=f
$$

The following decomposition is straightforward.

LEMMA 2.6. $\ell_{p}\left(h \mathbb{Z}^{n-1}\right)=h_{p}^{+} \oplus \bar{h}_{p}^{+}=h_{p}^{-} \oplus \bar{h}_{p}^{-}, \quad 1 \leq p<\infty$.

Proof. Immediate, since $P^{+}, Q^{+}$are projectors with $P^{+}+Q^{+}=I$ and $P^{+} Q^{+}=Q^{+} P^{+}=0$. Similar for $P^{-}, Q^{-}$.

Moreover, we remark that when $h \rightarrow 0$ we get $P^{+}, Q^{-} \rightarrow P$ and $P^{-}, Q^{+} \rightarrow Q$, where $P, Q$ denote the continuous Hardy projectors on the upper half plane.

Also of importance is the connection between the 0- and 1-layers of a discrete monogenic function $f$ on the upper half space, respectively, between the 0 - and (-1)layers of a discrete monogenic function $f$ on the lower half space. For that, we resort again to their description in Fourier domain.

First of all, let us denote by $F^{j}$ the discrete Fourier transform of $f^{j}, j=-1,0,1$. Second, using the isomorphism $\mathbb{C}_{n}=\mathbb{C}_{n-1} \otimes_{\mathbb{R}} \mathbb{C}_{1}$ we decompose the resulting function into its components in the $1, e_{n}^{+}, e_{n}^{-}, e_{n}^{+} e_{n}^{-}$basis, that is

$$
\mathcal{F}_{h} f^{j}=F^{j}=F_{1}^{j}+e_{n}^{+} F_{2}^{j}+e_{n}^{-} F_{3}^{j}+e_{n}^{+} e_{n}^{-} F_{4}^{j}, \quad F_{s}^{j} \in \ell_{p}\left(h \mathbb{Z}^{n-1}, \mathbb{C}_{n-1}\right), \quad s=1,2,3,4 .
$$

In addition to this, we emphasize the upper (respectively lower) case by adding an extra upper index + , (respectively, - ). That is to say, $F^{+, 1}$ will denote the discrete Fourier transform of $f^{1}$ taken as (partial) boundary data for the upper half case. In accordance with these notations,

(I) If $\left(e_{n}^{-} f^{0}, e_{n}^{+} f^{1}\right)$ is boundary data of a discrete upper monogenic function $f \in \ell_{p}\left(\mathbb{Z}_{+, 0}^{n}\right)$, then

(i) the components $F^{+, 1}=\mathcal{F}_{h} f^{1}$ satisfy to

$$
\left\{\begin{array}{c}
\frac{h \underline{d}-\sqrt{4+h^{2} \underline{d}^{2}}}{2} F_{1}^{+, 1}+\frac{\underline{\xi^{D}}}{\underline{\underline{d}}} F_{2}^{+, 1}=0 \\
\frac{h \underline{d}-\sqrt{4+h^{2} \underline{d}^{2}}}{2} F_{3}^{+, 1}+\frac{\underline{\underline{\xi}}}{\underline{\underline{d}}}\left(F_{1}^{+, 1}-F_{4}^{+, 1}\right)=0
\end{array}\right.
$$

(ii) by [6] - relation (36), it is proved that $F^{+, 1}$ can be uniquely writen in 
terms of the values of $F^{+, 0}=\mathcal{F}_{h} f^{0}$. Namely, we get

$$
\left\{\begin{array}{c}
F_{1}^{+, 1}=\frac{\underline{\underline{\xi}}}{\underline{d}}\left(\frac{\sqrt{4+h^{2} \underline{d^{2}}}-h \underline{d}}{2}\right) F_{2}^{+, 0} \\
F_{2}^{+, 1}=\frac{\sqrt{4+h^{2} \underline{d}^{2}}-h \underline{\underline{d}}}{\sqrt{4+h^{2} \underline{d}^{2}}+h \underline{d}} F_{2}^{+, 0} \\
F_{3}^{+, 1}=\frac{\underline{\xi}}{\underline{\underline{d}}}\left(\frac{\sqrt{4+h^{2} \underline{d^{2}}}-h \underline{d}}{2}\right)\left(F_{1}^{+, 0}-F_{4}^{+, 0}\right) \\
F_{4}^{+, 1}=\frac{\underline{\xi}}{\underline{\underline{d}}}\left(\frac{\sqrt{4+h^{2} \underline{d}^{2}}-h \underline{d}}{2}\right) F_{2}^{+, 0}-\frac{\sqrt{4+h^{2} \underline{d}^{2}}-h \underline{d}}{\sqrt{4+h^{2} \underline{d}^{2}}+h \underline{d}}\left(F_{1}^{+, 0}-F_{4}^{+, 0}\right)
\end{array}\right.
$$

(II) In a similar way, if $\left(e_{n}^{+} f^{0}, e_{n}^{-} f^{-1}\right)$ is boundary data of a discrete lower monogenic function $f \in \ell_{p}\left(\mathbb{Z}_{-, 0}^{n}\right)$, then

(i) $F^{-,-1}=\mathcal{F}_{h} f^{-1}$ satisfy

$$
\left\{\begin{array}{c}
\frac{h \underline{d}-\sqrt{4+h^{2} \underline{d}^{2}}}{2} F_{2}^{-,-1}-\frac{\underline{\xi^{D}}}{\underline{\underline{d}}} F_{1}^{-,-1}=0 \\
\frac{h \bar{d}-\sqrt{4+h^{2} \underline{d}^{2}}}{2}\left(F_{1}^{-,-1}-F_{4}^{-,-1}\right)-\frac{\underline{\underline{\xi}}}{\underline{\underline{d}}} F_{3}^{-,-1}=0
\end{array}\right.
$$

(ii) by [6] - relation (40), $F^{-,-1}$ can be uniquely writen in terms of the values of $F^{-, 0}=\mathcal{F}_{h} f^{0}$ as

$$
\left\{\begin{array}{c}
F_{1}^{-,-1}=\frac{\sqrt{4+h^{2} \underline{d}^{2}}-h \underline{d}}{\sqrt{4+h^{2} \underline{d}^{2}}+h \underline{d}} F_{1}^{-, 0} \\
F_{2}^{-,-1}=-\frac{\underline{\xi}}{\underline{\underline{d}}}\left(\frac{\sqrt{4+h^{2} \underline{d}^{2}}-h \underline{d}}{2}\right) F_{1}^{-, 0} \\
F_{3}^{-,-1}=\frac{\sqrt{4+h^{2} \underline{d}^{2}}-h \underline{d}}{\sqrt{4+h^{2} \underline{d}^{2}}+h \underline{d}} F_{3}^{-, 0} \\
F_{4}^{-,-1}=\frac{\sqrt{4+h^{2} \underline{d^{2}}}-h \underline{d}}{\sqrt{4+h^{2} \underline{d}^{2}}+h \underline{d}} F_{1}^{-, 0}+\frac{\underline{\underline{\xi}}}{\underline{\underline{d}}}\left(\frac{\sqrt{4+h^{2} \underline{d}^{2}}-h \underline{d}}{2}\right) F_{3}^{-, 0} .
\end{array}\right.
$$

\section{Discrete Hilbert BVP}

We aim to solve discrete Hilbert problems in higher dimensions. In the following sections, we will considered three particular types of such problems. However, before we start two observations must be made: the first is the fact that boundary data in the discrete setting depends on three distinct layers: two layers for the inner boundary and another two for the outer boundary (with the 0-layer in common). The second observation is the fact that whenever the boundary data belongs to a monogenic function then it is possible to relate the values from the two associated layers. Hence, we start with the explicit calculation of such relations. From these, we establish the upper and lower trace operators which, in turn, allow us to relate the Hardy projections with the discrete Cauchy transforms. 


\subsection{Boundary data relations}

For the boundary behaviour of a discrete monogenic function in the layer $m_{n}=1$ (resp. $m_{n}=-1$ ) we have in the Fourier domain (cf. [6] - relations (31) and (32), generalized to dimension $n$ )

$$
\begin{aligned}
& {\left[\frac{\underline{\underline{\xi}}}{\underline{d}}\left(\frac{2+h^{2} \underline{d}^{2}}{2 \sqrt{4+h^{2} \underline{d}^{2}}}-\frac{h \underline{d}}{2}\right)+e_{n}^{-}\left(\frac{1}{2}-\frac{h \underline{d}}{2 \sqrt{4+h^{2} \underline{d}^{2}}}\right)\right] e_{n}^{+} F^{+, 1}(\underline{\xi})+F^{+, 1}(\underline{\xi})} \\
& \quad=-\left[\frac{\underline{\xi}}{\underline{d}} \frac{1}{\sqrt{4+h^{2} \underline{d}^{2}}}+e_{n}^{+}\left(\frac{1}{2}-\frac{h \underline{d}}{2 \sqrt{4+h^{2} \underline{d}^{2}}}\right)\right] e_{n}^{-} F^{+, 0}(\underline{\xi}),
\end{aligned}
$$

as well as

$$
\begin{aligned}
& {\left[\frac{\underline{\underline{\xi}}}{\underline{d}}\left(\frac{2+h^{2} \underline{d}^{2}}{2 \sqrt{4+h^{2} \underline{d}^{2}}}-\frac{h \underline{d}}{2}\right)-e_{n}^{+}\left(\frac{1}{2}-\frac{h \underline{d}}{2 \sqrt{4+h^{2} \underline{d}^{2}}}\right)\right] e_{n}^{-} F^{-,-1}(\underline{\xi})-F^{-,-1}(\underline{\xi})} \\
& \quad=-\left[\frac{\underline{\xi}}{\underline{d}} \frac{1}{\sqrt{4+h^{2} \underline{d}^{2}}}-e_{n}^{-}\left(\frac{1}{2}-\frac{h \underline{d}}{2 \sqrt{4+h^{2} \underline{d}^{2}}}\right)\right] e_{n}^{+} F^{-, 0}(\underline{\xi}),
\end{aligned}
$$

where

$$
\underline{\xi} \in\left[-\frac{\pi}{h}, \frac{\pi}{h}\right]^{n-1}
$$

Now, the question of solvability of both (14) and (15) remains. We start with (14). First, we observe that

$$
C=\left[\frac{\underline{\xi}^{D}}{\underline{d}}\left(\frac{2+h^{2} \underline{d}^{2}}{2 \sqrt{4+h^{2} \underline{d}^{2}}}-\frac{h \underline{d}}{2}\right)+e_{n}^{-}\left(\frac{1}{2}-\frac{h \underline{d}}{2 \sqrt{4+h^{2} \underline{d}^{2}}}\right)\right] e_{n}^{+}+1
$$

is not invertible but has a left inverse given by

$$
\begin{aligned}
C_{l}^{-1}= & \frac{2 \sqrt{4+h^{2} \underline{d}^{2}}}{h \underline{d}+\sqrt{4+h^{2} \underline{d}^{2}}}-\frac{\underline{\xi}^{D}}{\bar{d}}\left(\frac{2+h^{2} \underline{d}^{2}-h \underline{d} \sqrt{4+h^{2} \underline{d}^{2}}}{h \underline{d}+\sqrt{4+h^{2} \underline{d}^{2}}}\right) e_{n}^{+} \\
& -\left(\frac{h \underline{d}-\sqrt{4+h^{2} \underline{d}^{2}}}{h \underline{d}+\sqrt{4+h^{2} \underline{d}^{2}}}\right) e_{n}^{+} e_{n}^{-} .
\end{aligned}
$$

Hereby, we recall that $\xi^{D} e_{n}^{ \pm}=-e_{n}^{ \pm} \xi^{D},\left(e_{n}^{ \pm}\right)^{2}=0, e_{n}^{-} e_{n}^{+} e_{n}^{-}=\left(-1-e_{n}^{+} e_{n}^{-}\right) e_{n}^{-}=-e_{n}^{-}$, and $\left(\xi^{D}\right)^{2}=\underline{d}^{2}$.

Hence, we get the equation relating the values of the function on the 1-layer with the function values on the 0-layer as

$$
F^{+, 1}(\underline{\xi})=\left[-\frac{\underline{\xi}^{D}}{\underline{d}}\left(\frac{2}{h \underline{d}+\sqrt{4+h^{2} \underline{d}^{2}}}\right)-e_{n}^{+}\right] e_{n}^{-} F^{+, 0}(\underline{\xi}) .
$$

In a similar way, we need to solve Equation (15) for the connection between layers -1 and 0 . 
Again, the term

$$
\tilde{C}=\left[\frac{\underline{\underline{\xi}}}{\underline{d}}\left(\frac{2+h^{2} \underline{d}^{2}}{2 \sqrt{4+h^{2} \underline{d}^{2}}}-\frac{h \underline{d}}{2}\right)-e_{n}^{+}\left(\frac{1}{2}-\frac{h \underline{d}}{2 \sqrt{4+h^{2} \underline{d}^{2}}}\right)\right] e_{n}^{-}-1
$$

has a left inverse given by

$$
\begin{aligned}
\tilde{C}_{l}^{-1}= & -\frac{2 \sqrt{4+h^{2} \underline{d}^{2}}}{h \underline{d}+\sqrt{4+h^{2} \underline{d}^{2}}}-\frac{\underline{\xi}^{D}}{\underline{d}}\left(\frac{2+h^{2} \underline{d}^{2}-h \underline{d} \sqrt{4+h^{2} \underline{d}^{2}}}{h \underline{d}+\sqrt{4+h^{2} \underline{d}^{2}}}\right) e_{n}^{-} \\
& +\left(\frac{h \underline{d}-\sqrt{4+h^{2} \underline{d}^{2}}}{h \underline{d}+\sqrt{4+h^{2} \underline{d}^{2}}}\right) e_{n}^{-} e_{n}^{+} .
\end{aligned}
$$

Hence, the connection between the values of the -1 - and the 0-layers is now

$$
F^{-,-1}(\underline{\xi})=\left[\frac{\underline{\xi}^{D}}{\underline{d}}\left(\frac{2}{h \underline{d}+\sqrt{4+h^{2} \underline{d}^{2}}}\right)-e_{n}^{-}\right] e_{n}^{+} F^{-, 0}(\underline{\xi}) .
$$

One remark must be made with respect to Equations (16) and (17). Since in both cases the boundary data at the 0-layer is multiplied by a zero divisor one has $\left(\mathrm{e}_{n}^{-} \mathrm{e}_{n}^{+} \mathrm{e}_{n}^{-}=-\mathrm{e}_{n}^{-}\right)$

$$
e_{n}^{-} F^{+, 0}=e_{n}^{-}\left[F_{1}^{+, 0}-F_{4}^{+, 0}+\mathrm{e}_{n}^{+} F_{2}^{+, 0}\right] \quad \text { and } \quad e_{n}^{+} F^{-, 0}=e_{n}^{+}\left[F_{1}^{-, 0}+\mathrm{e}_{n}^{-} F_{3}^{-, 0}\right] .
$$

Hence, the term $e_{n}^{-} F^{+, 0}=e_{n}^{-} \mathcal{F}_{h} f^{0}$ depends only on the components $F_{1}^{+, 0}-F_{4}^{+, 0}$ and $F_{2}^{+, 0}$. They are obtainable from system (11) by

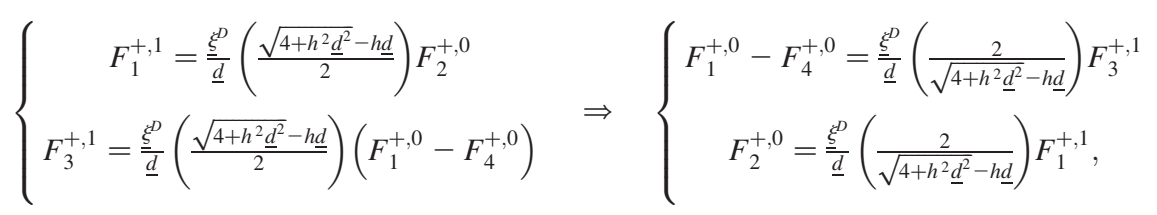

where $\sqrt{4+h^{2} \underline{d}^{2}}-h \underline{d}$ never vanishes. In addition, system (11) also ensures that the obtained boundary data is associated with a discrete monogenic function on $h \mathbb{Z}_{+}^{n}$. Therefore,

$$
\begin{aligned}
\mathcal{F}_{h} & \left(e_{n}^{-} f^{0}\right)=e_{n}^{-} \mathcal{F}_{h} f^{0}=e_{n}^{-} F^{+, 0}=e_{n}^{-}\left[\left(F_{1}^{+, 0}-F_{4}^{+, 0}\right)+\mathrm{e}_{n}^{+} F_{2}^{+, 0}\right] \\
= & e_{n}^{-}\left[\frac{2}{\underline{\underline{\xi}}}\left(\frac{2}{\sqrt{4+h^{2} \underline{d}^{2}}-h \underline{d}}\right) F_{3}^{+, 1}+e_{n}^{+} \frac{\underline{\underline{\xi}}}{\underline{d}}\left(\frac{2}{\sqrt{4+h^{2} \underline{d}^{2}}-h \underline{d}}\right) F_{1}^{+, 1}\right] \\
= & \frac{\underline{\xi}^{D}}{\underline{d}}\left(\frac{2}{\sqrt{4+h^{2} \underline{d}^{2}}-h \underline{d}}\right)\left[-e_{n}^{-} F_{3}^{+, 1}+e_{n}^{-} e_{n}^{+} F_{1}^{+, 1}\right] \\
= & \frac{2}{\underline{\underline{d}}}\left(\frac{2}{\sqrt{4+h^{2} \underline{d}^{2}}-h \underline{d}}\right) e_{n}^{-} e_{n}^{+}\left[F_{1}^{+, 1}+e_{n}^{+} F_{2}^{+, 1}+e_{n}^{-} F_{3}^{+, 1}+e_{n}^{+} e_{n}^{-} F_{4}^{+, 1}\right] .
\end{aligned}
$$


Hence, we have

$$
\begin{aligned}
e_{n}^{-} f^{0} & =\mathcal{F}_{h}^{-1}\left(\frac{\underline{\underline{\xi}}}{\underline{d}}\left(\frac{2}{\sqrt{4+h^{2} \underline{d}^{2}}-h \underline{d}}\right) e_{n}^{-} e_{n}^{+}\left[F_{1}^{+, 1}+e_{n}^{+} F_{2}^{+, 1}+e_{n}^{-} F_{3}^{+, 1}+e_{n}^{+} e_{n}^{-} F_{4}^{+, 1}\right]\right) \\
= & : e_{n}^{-} \mathscr{A}_{+}\left[-e_{n}^{+} f^{1}\right] .
\end{aligned}
$$

Analogous, for the lower case the term $e_{n}^{+} F^{-, 0}=e_{n}^{+} \mathcal{F}_{h} f^{0}$ depends only on the components $F_{1}^{-, 0}$ and $F_{3}^{-, 0}$. From system (13) we get

$$
\left\{\begin{array} { l } 
{ F _ { 1 } ^ { - , - 1 } = ( \frac { \sqrt { 4 + h ^ { 2 } \underline { d } ^ { 2 } } - h \underline { d } } { \sqrt { 4 + h ^ { 2 } \underline { d } ^ { 2 } } + h \underline { d } } ) F _ { 1 } ^ { - , 0 } } \\
{ F _ { 3 } ^ { - , - 1 } = ( \frac { \sqrt { 4 + h ^ { 2 } \underline { d } ^ { 2 } } - h \underline { d } } { \sqrt { 4 + h ^ { 2 } \underline { d } ^ { 2 } } + h \underline { d } } ) F _ { 3 } ^ { - , 0 } }
\end{array} \Rightarrow \left\{\begin{array}{l}
F_{1}^{-, 0}=\left(\frac{\sqrt{4+h^{2} \underline{d}^{2}}+h \underline{d}}{\sqrt{4+h^{2} \underline{d}^{2}}-h \underline{d}}\right) F_{1}^{-,-1} \\
F_{3}^{-, 0}=\left(\frac{\sqrt{4+h^{2} \underline{d}^{2}}+h \underline{d}}{\sqrt{4+h^{2} \underline{d}^{2}}-h \underline{d}}\right) F_{3}^{-,-1}
\end{array}\right.\right.
$$

Then,

$$
\begin{aligned}
\mathcal{F}_{h}\left(e_{n}^{+} f^{0}\right)=e_{n}^{+} \mathcal{F}_{h} f^{0}=e_{n}^{+} F^{-, 0}=e_{n}^{+}\left[\left(F_{1}^{-, 0}+\mathrm{e}_{n}^{-} F_{3}^{-, 0}\right]\right. \\
=e_{n}^{+}\left[\left(\frac{\sqrt{4+h^{2} \underline{d}^{2}}+h \underline{d}}{\sqrt{4+h^{2} \underline{d}^{2}}-h \underline{d}}\right)\left(F_{1}^{-,-1}+e_{n}^{-} F_{3}^{-,-1}\right) \cdot\right]=: e_{n}^{+} \mathscr{A}_{-}\left[F^{-,-1}\right] .
\end{aligned}
$$

which results in

$$
\begin{aligned}
e_{n}^{+} f^{0} & =\mathcal{F}_{h}^{-1}\left(e_{n}^{+}\left[\left(\frac{\sqrt{4+h^{2} \underline{d}^{2}}+h \underline{d}}{\sqrt{4+h^{2} \underline{d}^{2}}-h \underline{d}}\right)\left(F_{1}^{-,-1}+e_{n}^{-} F_{3}^{-,-1}\right) \cdot\right]\right) \\
= & : e_{n}^{+} \mathscr{A}_{-}\left[f^{-1}\right] .
\end{aligned}
$$

In the above calculations appear certain terms which we designated by $\mathscr{A}_{+}$and $\mathscr{A}_{-}$. Since we are going to use them later we are going to give an explicit definition.

DEFINITION 3.1. We define the operators $\mathscr{A}_{+}$and $\mathscr{A}_{-}$, respectively, as

(i) $\mathscr{A}_{+}: \ell_{p}\left(h \mathbb{Z}^{n-1}\right) \rightarrow \ell_{p}\left(h \mathbb{Z}^{n-1}\right)$, given by

$$
\mathscr{A}_{+}[f]:=\mathcal{F}_{h}^{-1}\left[\frac{\underline{\xi}^{D}}{\underline{d}}\left(\frac{2}{\sqrt{4+h^{2} \underline{d}^{2}}-h \underline{d}}\right) \mathcal{F}_{h} f\right]
$$

(ii) $\mathscr{A}_{-}: \ell_{p}\left(h \mathbb{Z}^{n-1}\right) \rightarrow \ell_{p}\left(h \mathbb{Z}^{n-1}\right)$, given by

$$
\mathscr{A}_{-}[f]:=\mathcal{F}_{h}^{-1}\left[\left(\frac{\sqrt{4+h^{2} \underline{d}^{2}}+h \underline{d}}{\sqrt{4+h^{2} \underline{d}^{2}}-h \underline{d}}\right) \mathcal{F}_{h} f\right] .
$$


Due to the construction these operators characterize the 0-layer values of upper/lower discrete monogenic functions.

These considerations mean that the values on the 1-layer or the -1-layer are enough to describe our discrete monogenic functions and, consequently, we propose the following definition of a discrete trace operator.

Definition 3.2. (Upper and lower trace operator). Given $f \in \ell_{p}\left(h \mathbb{Z}^{n}\right)$, we define the

(i) upper trace operator $\operatorname{tr}_{+}: \ell_{\mathrm{p}}\left(\mathrm{h} \mathbb{Z}^{\mathrm{n}}\right) \rightarrow \ell_{\mathrm{p}}\left(\mathrm{h} \mathbb{Z}^{\mathrm{n}-1}\right) \times \ell_{\mathrm{p}}\left(\mathrm{h} \mathbb{Z}^{\mathrm{n}-1}\right)$ as

$$
\operatorname{tr}_{+}[f]:=\left(e_{n}^{-} \mathscr{A}_{+}\left[-\mathrm{e}_{\mathrm{n}}^{+} f^{1}\right], e_{n}^{+} f^{1}\right),
$$

with $f^{1}(h \underline{m}):=f(h(\underline{m}, 1))$.

(ii) lower trace operator $\operatorname{tr}_{-}: \ell_{p}\left(h \mathbb{Z}^{n}\right) \rightarrow \ell_{p}\left(h \mathbb{Z}^{n-1}\right) \times \ell_{p}\left(h \mathbb{Z}^{n-1}\right)$ as

$$
\operatorname{tr}_{-}[f]:=\left(e_{n}^{+} \mathscr{A}_{-}\left[f^{-1}\right], e_{n}^{-} f^{-1}\right),
$$

with $f^{-1}(h \underline{m}):=f(h(\underline{m},-1))$.

The upper/lower trace operators generate a pair of boundary data which can be monogenically extended by the Cauchy transform to the upper/lower half lattice. In particular, we get the following discrete version of the projection properties of the trace of the Cauchy transform.

Lemma 3.3. Let $f \in \ell_{p}\left(h \mathbb{Z}^{n}\right)$. Then

(i) $C^{+} \operatorname{tr}_{+}\left[\mathrm{C}^{+} \operatorname{tr}_{+}[f]\right]=\mathrm{C}^{+} \operatorname{tr}_{+}[f]$;

(ii) $C^{-} \operatorname{tr}_{-}\left[\mathrm{C}^{-} \operatorname{tr}_{-}[f]\right]=\mathrm{C}^{-} \operatorname{tr}_{-}[f]$;

As we can see in the above lemma $C^{+} \operatorname{tr}_{+}$and $C^{-} \operatorname{tr}$ - project the function $f$ into the space of functions which can be monogenically extended to the upper half space and the lower half space, respectively.

The upper/lower trace operator acts on a function defined in the upper/lower half space. However, we have also to consider the case when the function is given only on either the 1- or -1-layer, respectively. For this propose we introduce the upper and lower boundary generators.

DeFINITION 3.4. (Upper and lower boundary generators). Given $g \in \ell_{p}\left(h \mathbb{Z}^{n-1}\right)$ we define the

(1) upper boundary generator $\mathcal{G}_{+}: \ell_{p}\left(h \mathbb{Z}^{n-1}\right) \rightarrow \ell_{p}\left(h \mathbb{Z}^{n-1}\right) \times \ell_{p}\left(h \mathbb{Z}^{n-1}\right)$ as

$$
\mathcal{G}_{+}[g]:=\left(e_{n}^{-} \mathscr{A}_{+}\left[-e_{n}^{+} g\right], e_{n}^{+} g\right) .
$$

(2) lower boundary generator $\mathcal{G}_{-}: \ell_{p}\left(h \mathbb{Z}^{n-1}\right) \rightarrow \ell_{p}\left(h \mathbb{Z}^{n-1}\right) \times \ell_{p}\left(h \mathbb{Z}^{n-1}\right)$ as

$$
\mathcal{G}_{-}[g]:=\left(e_{n}^{+} \mathscr{A}_{-}[g], e_{n}^{-} g\right) \text {. }
$$

Let us remark that the above definition of boundary generators is something particular to the discrete case. In the limit $h \rightarrow 0$ these operators converge to the identity operator and 
one does not make a real distinction between the boundary data $\mathcal{G}_{+}[g]$ and the boundary function $g$.

Obviously, we have that $\operatorname{tr}_{+}[f]=\mathcal{G}_{+}\left[f^{1}\right]$, where $f^{1}(h \underline{m}):=f(h(\underline{m}, 1))$. Analogous for the lower case.

Moreover, from the above construction we obtain the following description for the Hardy projectors.

THEOREM 3.5. Let $g \in \ell_{p}\left(h \mathbb{Z}^{n-1}\right)$. Then one gets

$$
P_{+} g(h \underline{m})=C^{+} \mathcal{G}_{+}[g](h(\underline{m}, 1)), \quad P_{-} g(h \underline{m})=C^{-} \mathcal{G}_{-}[g](h(\underline{m},-1)),
$$

for all $\underline{m} \in h \mathbb{Z}^{n-1}$.

Proof. As the two statements are similar, we prove only the upper case. Given a function $g \in \ell_{p}\left(h \mathbb{Z}^{n-1}\right)$, we have $\mathcal{G}_{+}[g]=\left(e_{n}^{-} \mathscr{A}_{+}\left[-e_{n}^{+} g\right], e_{n}^{+} g\right)$.

Let $f:=C^{+} \mathcal{G}_{+}[g]$. Then, its restriction to the 1-layer, $f^{1}(h \underline{m}):=f(h(\underline{m}, 1))$, satisfy

$$
e_{n}^{+} g=e_{n}^{+} f^{1} \quad \text { and } \quad \mathcal{G}_{+}\left[f^{1}\right]=\left(e_{n}^{-} \mathscr{A}_{+}\left[-e_{n}^{+} f^{1}\right], e_{n}^{+} f^{1}\right)=\mathcal{G}_{+}[g] .
$$

Hence,

$$
C^{+} \mathcal{G}_{+}[g]=C^{+} \mathcal{G}_{+}\left[f^{1}\right]=C^{+} \mathcal{G}_{+}\left[C^{+} \mathcal{G}_{+}[g](h(\cdot, 1))\right]=\left(C^{+} \mathcal{G}_{+}\right)^{2}[g],
$$

that is to say, $C^{+} \mathcal{G}_{+}$is a projector, and $C^{+} \mathcal{G}_{+}[g](h(\cdot, 1))=P^{+}[g]$.

\subsection{Boundary value problems}

As a starting point we consider the Hilbert problem of reconstructing a monogenic function in the discrete upper half plane from its boundary data.

Problem I. Given $g \in \ell_{p}\left(h \mathbb{Z}^{n-1}\right),(1 \leq p<+\infty)$, we want to determine $f: h \mathbb{Z}_{+}^{n} \rightarrow \mathbb{C}_{n}$ such that

$$
\left\{\begin{array}{cc}
D_{h}^{+-} f(h m)=0, & m \in \mathbb{Z}_{+}^{n}, \\
f(h(\underline{m}, 1))=g(h \underline{m}), & \underline{m} \in \mathbb{Z}^{n-1} .
\end{array}\right.
$$

As in the continuous case this problem has an almost immediate solution.

THEOREM 3.6. The boundary value problem (21) is uniquely solvable if and only if the (partial) boundary data $g$ is in $h_{p}^{+}$.

Moreover, its solution is given by

$$
f(h m)=C^{+} \mathcal{G}_{+}[g](h m),
$$

for $m=\left(\underline{m}, m_{n}\right) \in \mathbb{Z}^{n-1} \times \mathbb{Z}_{+}$. 
Proof. Assume that $g \notin h_{p}^{+}$. Obviously, a discrete monogenic function $f$ such that its values on the 1-layer fulfil the given boundary condition does not exist. Therefore, $g \in h_{p}^{+}$ is a necessary condition for the existence of a solution to problem (21).

Next, we prove that a solution exists in this case. Given $g \in h_{p}^{+}$one applies the boundary generator, i.e.

$$
\mathcal{G}_{+}[g]=\left(e_{n}^{-} \mathscr{A}_{+}\left[-e_{n}^{+} g\right], e_{n}^{+} g\right)
$$

Hence, we have that $f=C^{+} \mathcal{G}_{+}[g]$ is a discrete monogenic function on the upper half lattice satisfying to $f(h(\underline{m}, 1))=g(\underline{m})$. Its uniqueness is guaranteed by the maximum principle (cf. [6], Corollary 2.12).

COROLlaRY 3.7. A similar result holds for the corresponding problem in the lower half lattice $\mathbb{Z}_{-}^{n}$, with $g \in h_{p}^{-}$, where its unique solution is given by

$$
f(h m)=C^{-} \mathcal{G}_{-}[g](h m), \quad m=\left(\underline{m}, m_{n}\right) \in \mathbb{Z}^{n-1} \times \mathbb{Z}_{-} .
$$

\section{Remark 3.8.}

(I) In Theorem 3.6 the boundary value problem is studied for a given partial boundary data on the 1-layer. Obviously, a similar discussion could be made for a partial boundary data given on the 0-layer. However, as $C^{+}\left[e_{n}^{-} f^{0}, e_{n}^{+} f^{1}\right](\underline{m}, 0)=0$ for any boundary data $\left(e_{n}^{-} f^{0}, e_{n}^{+} f^{1}\right)$ of an upper discrete monogenic function $f$ this problem must be stated in terms not of the values of the resulting function on the 0-layer, but in terms of the first component of its trace operator. An analogous remark holds for Corollary 3.7.

(II) When $n=2$ and $h$ tends to 0 then, problem (21) reduces to the Hilbert boundary value problem for analytic functions on the upper half of the complex plane. We remark that this particular type of Hilbert boundary value problems were already discussed in $[7,19]$.

\subsection{Jump problem 1}

Let us formulate the discrete equivalent to the classic Hilbert boundary value problem. Due to the fact that in the discrete case the boundary actually consists of three interlinked layers one has a certain freedom in imposing jump conditions, a freedom which does not exist in the continuous case. Let us start with the case in which the jump condition is given in terms of values on the 0-layer. Due to Theorem 2.2 a discrete monogenic function on $h \mathbb{Z}^{n} \backslash\left\{m_{n}=0\right\}$ takes the value zero on the 0-layer. Therefore, a jump condition on the 0 layer only makes sense when it is assumed as the difference between the first components of the upper and the lower traces of the function. 
Problem II. Given a function $g \in \ell_{p}\left(h \mathbb{Z}^{n-1}\right),(1 \leq p<n)$, we want to determine a discrete monogenic $f: h \mathbb{Z}^{n} \rightarrow \mathbb{C}_{n}$ subjected to a jump condition, that is

$$
\left\{\begin{array}{cc}
D_{h}^{+-} f(h m)=0, & m \in \mathbb{Z}^{n} \backslash\left\{m_{n}=0\right\}, \\
e_{n}^{-} f_{+}(h \underline{m}, 0)-e_{n}^{+} f_{-}(h \underline{m}, 0)=e_{n} g(h \underline{m}), & \underline{m} \in \mathbb{Z}^{n-1} .
\end{array}\right.
$$

For the solvabilty of this problem we can state the following theorem.

THEOREM 3.9. For an arbitrary function $g=g^{1}+e_{n}^{+} g^{2}+e_{n}^{-} g^{3}+e_{n}^{+} e_{n}^{-} g^{4} \in$ $\ell_{p}\left(\mathbb{Z}^{n-1}\right),(1 \leq p<n)$, the Hilbert boundary value problem with jump condition

$$
\left\{\begin{array}{cc}
D_{h}^{+-} f(h m)=0, & m \in \mathbb{Z}^{n} \backslash\left\{m_{n}=0\right\}, \\
e_{n}^{-} f_{+}(h \underline{m}, 0)-e_{n}^{+} f_{-}(h \underline{m}, 0)=e_{n} g(h \underline{m}), & \underline{m} \in \mathbb{Z}^{n-1},
\end{array}\right.
$$

is uniquely solvable with

$$
f(h m)=\left\{\begin{array}{cc}
C^{+} \mathcal{G}_{+}\left[g_{+}\right](h m), & m_{n} \geq+1 \\
C^{-} \mathcal{G}_{-}\left[-g_{-}\right](h m), & m_{n} \leq-1
\end{array}\right.
$$

where $g_{-}:=g^{1}+e_{n}^{-} g^{3}, g_{+}:=g^{1}-g^{4}+e_{n}^{+} g^{2}$.

Proof. First, we observe that $e_{n}$ is invertible. Moreover,

$$
\begin{aligned}
e_{n} g & =\left(e_{n}^{+}+e_{n}^{-}\right)\left(g^{1}+e_{n}^{+} g^{2}+e_{n}^{-} g^{3}+e_{n}^{+} e_{n}^{-} g^{4}\right) \\
& =e_{n}^{+}\left(g^{1}+e_{n}^{-} g^{3}\right)+e_{n}^{-}\left(g^{1}+e_{n}^{+} g^{2}-g^{4}\right):=e_{n}^{+} g_{-}+e_{n}^{-} g_{+} .
\end{aligned}
$$

Hence, we get as first components of the upper, resp. lower, trace of $f$

$$
e_{n}^{-} f^{+, 0}:=e_{n}^{-}\left(g^{1}+e_{n}^{+} g^{2}-g^{4}\right)=e_{n}^{-} g_{+}, \quad e^{+} f^{-, 0}:=-e_{n}^{+}\left(g^{1}+e_{n}^{-} g^{3}\right)=-e_{n}^{+} g_{-} .
$$

By relations (11) and (13) we obtain the remaining values of the upper and lower traces, namely $e_{n}^{+} f^{+, 1}$ and $e_{n}^{-} f^{-,-1}$. Moreover, these traces coincide with $\mathcal{G}_{+}\left[g_{+}\right]$, and $\mathcal{G}_{-}\left[-g_{-}\right]$, resp. By applying the upper (resp., lower) Cauchy transform to these pairs we obtain the discrete monogenic function

$$
f(h m)=\left\{\begin{array}{cc}
C^{+} \mathcal{G}_{+}\left[g_{+}\right](h m), & m_{n} \geq+1 \\
C^{-} \mathcal{G}_{-}\left[-g_{-}\right](h m), & m_{n} \leq-1
\end{array}\right.
$$

which satisfies to

$$
\begin{aligned}
e_{n}^{-} f_{+}(h \underline{m}, 0)-e_{n}^{+} f_{-}(h \underline{m}, 0) & =e_{n}^{-} f^{+, 0}(h \underline{m}, 0)-e_{n}^{+} f^{-, 0}(h \underline{m}, 0) \\
= & e_{n}^{-} g_{+}(h \underline{m})+e_{n}^{+} g_{-}(h \underline{m})=e_{n} g(h \underline{m}) .
\end{aligned}
$$


The above considerations allow us to look now at the jump problem relating the boundary values in the 1 - and -1-layer.

Problem III. Given a function $g \in \ell_{p}\left(h \mathbb{Z}^{n-1}\right),(1 \leq p<n)$, we want to determine a discrete monogenic function $f: h \mathbb{Z}^{n} \rightarrow \mathbb{C}_{n}$ subject to the following jump condition

$$
\left\{\begin{array}{cc}
D_{h}^{+-} f(h m)=0, & m \in \mathbb{Z}^{n} \backslash\left\{m_{n}=0\right\}, \\
e_{n}^{-} \mathscr{A}_{+}[f](h(\underline{n}, 1))-e_{n}^{+} \mathscr{A}_{-}[f](h(\underline{n},-1))=g(h \underline{m}), & \underline{m} \in \mathbb{Z}^{n-1} .
\end{array}\right.
$$

Several interpretations are possible for this jump condition. Again, notice that since the boundary data is in fact given by a pair of functions the jump condition can be expressed either in terms of the values on the 1- and -1-layer of the discrete function $f$, or in terms of their connection at the 0 -layer. Here, we assume this link is given by the difference of their respective extensions to the 0-layer.

However, let us point out that in both cases as $h \rightarrow 0$ the functions $f_{+}$and $f_{-}$given in the layers will converge to the continuous boundary values from above, resp. below, of the continuous function $f$.

Let us take a closer look at the boundary condition $\mathscr{A}_{+}[f](h(\underline{n}, 1))-\mathscr{A}_{-}$ $[f](h(\underline{n},-1))=g(h \underline{m})$. Since $1=-e_{n}^{2}$ we can rewrite $g$ as

$$
g(h \underline{m})=-e_{n}^{2} g(h \underline{m})=e_{n}^{+}\left(-e_{n} g\right)+e_{n}^{-}\left(-e_{n} g\right) .
$$

Keep in mind that this algebraic decomposition is unique. Since both $\mathscr{A}_{+}$and $\mathscr{A}_{-}$are invertible with their inversion formulae being given by (11) and (13), respectively, we can take $e_{n}^{+} f^{0}=e_{n}^{+}\left(-e_{n} g\right)$ and $e_{n}^{+} f^{0}=e_{n}^{-}\left(-e_{n} g\right)$. From this we get

$$
e_{n}^{+} f^{-1}=e_{n}^{+} \mathscr{A}_{-}^{-1}\left[e_{n} g\right] \text { and } e_{n}^{-} f^{1}=e_{n}^{-} \mathscr{A}_{+}^{-1}\left[-e_{n} g\right] .
$$

By applying now the Cauchy transform we arrive at the following theorem.

THEOREM 3.10. For an arbitrary function $g \in \ell_{p}\left(h \mathbb{Z}^{n-1}\right),(1 \leq p<n)$, the Hilbert boundary value problem with jump condition

$$
\left\{\begin{array}{cc}
D_{h}^{+-} f(h m)=0, & m \in \mathbb{Z}^{n} \backslash\left\{m_{n}=0\right\}, \\
e_{n}^{-} \mathscr{A}_{+}[f](h(\underline{n}, 1))-e_{n}^{+} \mathscr{A}_{-}[f](h(\underline{n},-1))=g(h \underline{m}), & \underline{m} \in \mathbb{Z}^{n-1} .
\end{array}\right.
$$

has an unique solution given by

$$
f(h m)=\left\{\begin{array}{cc}
C^{+}\left[e_{n}^{+} e_{n} g, e_{n}^{-} \mathscr{A}_{+}^{-1}\left[-e_{n} g\right]\right](h m), & m_{n} \geq+1 \\
C^{-}\left[e_{n}^{-} e_{n} g, e_{n}^{+} \mathscr{A}_{-}^{-1}\left[e_{n} g\right]\right](h m), & m_{n} \leq-1
\end{array}\right.
$$

We end up this section with the remark that problems (26) and (24) are equivalent, as expected. Therefore, in the following Hilbert problems we shall consider only the case in which the jump condition is expressed in terms of the values of the function in the 1-and -1-layers. 


\subsection{Jump problem 2}

The previous problem can be easily extended to the following setting.

Problem IV. Given $g \in \ell_{p}\left(h \mathbb{Z}^{n-1}\right),(1 \leq p<n)$ and a constant $\lambda \in \mathbb{C}_{n}$ with a right inverse $\lambda_{r}^{-1}$, we want to find $f: h \mathbb{Z}^{n} \rightarrow \mathbb{C}_{n}$ such that

$$
\left\{\begin{array}{cc}
D_{h}^{+-} f(h m)=0, & m \in \mathbb{Z}^{n} \backslash\left\{m_{n}=0\right\}, \\
e_{n}^{-} \mathscr{A}_{+}[f](h(\underline{n}, 1))-e_{n}^{+} \mathscr{A}_{-}[f](h(\underline{n},-1)) \lambda=g(h \underline{m}), & \underline{m} \in \mathbb{Z}^{n-1} .
\end{array}\right.
$$

The solution is almost again immediate.

THEOREM 3.11. For an arbitrary $g \in \ell_{p}\left(h \mathbb{Z}^{n-1}\right),(1 \leq p<n)$, and $\lambda \in \mathbb{C}_{n}$ with a right inverse $\lambda_{r}^{-1}$, the boundary value problem (28) is uniquely solvable. It solution is given by

$$
f(h m)=\left\{\begin{array}{cc}
C^{+}\left[e_{n}^{+} e_{n} g, e_{n}^{-} \mathscr{A}_{+}^{-1}\left[-e_{n} g\right]\right](h m), & m_{n} \geq+1 \\
C^{-}\left[e_{n}^{-} e_{n} g, e_{n}^{+} \mathscr{A}_{-}^{-1}\left[e_{n} g \lambda_{r}^{-1}\right]\right](h m), & m_{n} \leq-1
\end{array}\right.
$$

Since the proof of this theorem is an adaptation of the one of Theorem 3.10 we shall omit it here. However, we have to point out that $\mathscr{A}_{-}^{-1}\left[e_{n} g \lambda_{r}^{-1}\right]$ belongs to $h_{p}^{-}$since this space is a right linear module.

\section{Convergence results}

While discrete Hilbert problems have direct applications (see, e.g. [7,18]), nevertheless they can also be considered as discretizations of continuous Hilbert problems. This leads to the question of convergence when the lattice constant $h(0<h<1)$ goes to zero. In this section, we are going to deal with this problem, i.e. we study the convergence of the previous discrete problems to their continuous counterparts.

\subsection{Convergence results for the Hilbert problems}

In order to fix notations, we recall the continuous Hilbert problem. Let $f \in L_{p}\left(\mathbb{R}_{+}^{n}\right),(1<p<+\infty)$, be a solution of the problem

$$
\left\{\begin{array}{cc}
D f(x)=0, & x \in \mathbb{R}_{+}^{n} \\
f_{+}(\underline{x})=g(\underline{x}), & \underline{x} \in \mathbb{R}^{n-1} .
\end{array}\right.
$$

Here, $f_{+}$denotes the non-tangential limit of $f$ when $x \in \mathbb{R}_{+}^{n}$ goes to $\underline{x} \in \mathbb{R}^{n-1}$. If $g$ belongs to the the continuous Hardy space $H_{p}^{+}$, that is to the space of all functions in $L_{p}\left(\mathbb{R}^{n-1}\right)$ which are boundary values of monogenic functions on $\mathbb{R}_{+}^{n}$ (see also $[16,20]$ ), then $f$ is given in terms of the continuous Cauchy transform

$$
f(x)=\mathcal{C}_{+}[g](x)=\int_{\mathbb{R}^{n-1}} E(\underline{y}-x)\left(-e_{n}\right) g(\underline{y}) d \Gamma_{\underline{y}}, \quad x \in \mathbb{R}_{+}^{n},
$$


where $E$ denotes the fundamental solution for the Dirac operator $D=\sum_{j=1}^{n} e_{j} \partial_{x_{j}}$. Hence, we first investigate the convergence of the discrete solution (22), i.e. $C^{+} \mathcal{G}_{+}[g](h m)$, to the continuous solution (31) restricted to the lattice. Since

$C^{+} \mathcal{G}_{+}[g](h m)=-\sum_{\underline{\eta} \in \mathbb{Z}^{n-1}}\left[E_{h}^{-+}\left(h\left(\underline{\eta}-\underline{m},-m_{n}\right)\right) e_{n}^{+} g(h \underline{\eta})+E_{h}^{-+}\left(h\left(\underline{\eta}-\underline{m}, 1-m_{n}\right)\right) e_{n}^{-} \mathscr{A}_{+}\left[-e_{n}^{+} g\right](h \underline{\eta})\right] h^{n-1}$,

we begin by decomposing the boundary out-normal in (31) into $-e_{n}=-\left(e_{n}^{+}+e_{n}^{-}\right)$.

Second, we remark that if $g \in L_{p}\left(\mathbb{R}^{n-1}, \mathbb{C}_{n}\right) \cap C^{\alpha}\left(\mathbb{R}^{n-1}, \mathbb{C}_{n}\right)$, where $0<\alpha \leq 1$ and $1<p<\infty$, then, we have (see [9])

$$
\left\|R_{h} g\right\|_{\ell_{p+(n-1 / \alpha)}} \leq C\|g\|_{L_{p}}
$$

thus, ensuring that the function $g$ has a meaningful $\ell_{p+(n-1 / \alpha)}$ projection after its restriction to the lattice. Hence forward, and whenever it is clear from the context, we will denote by $g$ both the function $g$ and its restriction $R_{h} g$ to the lattice.

Lemma 4.1. Let $g \in H_{p}^{+} \cap C^{\alpha}\left(\mathbb{R}^{n-1}, \mathbb{C}_{n}\right) \cap W_{p}^{1}\left(\mathbb{R}^{n-1}, \mathbb{C}_{n}\right)$, with $0<\alpha \leq 1,1<p<n$. Then, we have

$$
\begin{aligned}
& \left|\int_{\mathbb{R}^{n-1}} E(\underline{y}-h m)\left(-e_{n}^{+}\right) g(\underline{y}) d \Gamma_{\underline{y}}-\sum_{\underline{\eta} \in \mathbb{Z}^{n-1}} E_{h}^{-+}\left(h\left(\underline{\eta}-\underline{m},-m_{n}\right)\right)\left(-e_{n}^{+}\right) g(h \underline{\eta}) h^{n-1}\right| \\
& \quad \leq\left(A h^{n-1}+B h\right)\|g\|_{L_{p}},
\end{aligned}
$$

for all $h m \in h \mathbb{Z}_{+}^{n}$, where $A, B>0$ are constants independent of $h$ and $g$, and $(1 / p)+(1 / q)=1$.

Proof. The restriction of $g \in H_{p}^{+}$to the lattice does not necessarily belong to $h_{p}^{+}$. However, Theorem 3.5 ensures $C^{+} \mathcal{G}_{+}\left[Q^{+} g\right]=0$. Hence, $C^{+} \mathcal{G}_{+}[g]=C^{+} \mathcal{G}_{+}\left[\left(P_{+}+Q_{+}\right) g\right]=$ $C^{+} \mathcal{G}_{+}\left[P_{+} g\right]$, with $P_{+} g \in h_{p}^{+}$. 
Now, let $W(h \eta)$ be a hypercube on $\mathbb{R}^{n-1}$ centred in $h \eta$ and with size-length $h$. For an arbitrary $m \in \mathbb{Z}_{+}^{n-}$ we have

$$
\begin{aligned}
& \left|\int_{\mathbb{R}^{n-1}} E\left(\underline{y}-h \underline{m},-h m_{n}\right)\left(-e_{n}^{+}\right) g(\underline{y}) d \Gamma_{\underline{y}}-\sum_{\underline{\eta} \in \mathbb{Z}^{n-1}} E_{h}^{-+}\left(h\left(\underline{\eta}-\underline{m},-m_{n}\right)\right)\left(-e_{n}^{+}\right) g(h \underline{\eta}) h^{n-1}\right| \\
& \quad \leq\left|\sum_{\underline{\eta} \in \mathbb{Z}^{n-1}}\left(\int_{W(h \underline{\eta})} E\left(\underline{y}-h \underline{\underline{m}},-h m_{n}\right) e_{n}^{+} g(\underline{y}) d \Gamma_{\underline{y}}-E\left(h\left(\underline{\eta}-\underline{m},-m_{n}\right)\right) e_{n}^{+} g(h \underline{\eta}) h^{n-1}\right)\right| \\
& \quad+\left|\sum_{\underline{\eta} \in \mathbb{Z}^{n-1}}\left(\left(E\left(h\left(\underline{\eta}-\underline{m},-m_{n}\right)\right)\right)-E_{h}^{-+}\left(h\left(\underline{\eta}-\underline{m},-m_{n}\right)\right)\right) e_{n}^{+} g(\underline{h} \underline{\eta}) h^{n-1}\right| \\
& \quad \leq\left|\sum_{\underline{\eta} \in \mathbb{Z}^{n-1}}\left(\int_{W(h \underline{\eta})} E\left(\underline{y}-h \underline{m},-h m_{n}\right) e_{n}^{+} g(\underline{y}) d \underline{\underline{y}}-E\left(h\left(\underline{\eta}-\underline{m},-m_{n}\right)\right) e_{n}^{+} g(h \underline{\eta}) h^{n-1}\right)\right| \\
& \quad+c_{1}^{2} \sum_{\underline{\eta} \in \mathbb{Z}^{n-1}}\left|\left(E\left(h\left(\underline{\eta}-\underline{m},-m_{n}\right)\right)-E_{h}^{-+}\left(h\left(\underline{\eta}-\underline{m},-m_{n}\right)\right)\right)\right| g(h \underline{\eta}) \mid h^{n-1}
\end{aligned}
$$

with $c_{1}^{2}=2^{n+1 / 2}$. Now, we apply Hölder's inequality with $1 / s+1 /(p+(n-1 / \alpha))=1$ to this last term. This leads to

$$
\begin{aligned}
& \sum_{\underline{\eta} \in \mathbb{Z}^{n-1}}\left|E_{h}^{-+}\left(h\left(\underline{\eta}-\underline{m},-m_{n}\right)\right)-E\left(h\left(\underline{\eta}-\underline{m},-m_{n}\right)\right)\right||g(h \underline{\eta})| h^{n-1} \\
& \quad \leq\left(\sum_{\underline{\eta} \in \mathbb{Z}^{n-1}}\left|E_{h}^{-+}\left(h\left(\underline{\eta}-\underline{m},-m_{n}\right)\right)-E\left(h\left(\underline{\eta}-\underline{m},-m_{n}\right)\right)\right|^{s} h^{n-1}\right)^{1 / s}\|g\|_{\ell_{p+(n-1) / \alpha}} \\
& \quad \leq C_{1} h^{1+(n-1) / s}\|g\|_{\ell_{p+(n-1) / \alpha}}
\end{aligned}
$$

where $C_{1}>0$ is a constant independent of $h$ and of $g$ (cf. Lemma 2.8, [6]). Since $p+(n-1) / \alpha>1+(n-1)=n$ we get $1 / s>1-1 / n$ and, therefore, $1+(n-1) / s>$ $1+(n-1)(1-1 / n)=\left(n^{2}-n+1\right) / n>n-1$. This implies $h^{1+(n-1) / s}<h^{n-1}$ when $0<h<1$ and, therefore, (34) can be further estimated by

$$
C_{1} h^{1+(n-1) / s}\|g\|_{\ell_{p+(n-1) / \alpha}} \leq C_{1} h^{n-1}\|g\|_{L_{p}} .
$$


For the remaining term in (33) we have

$$
\begin{aligned}
& \left|\sum_{\underline{\eta} \in \mathbb{Z}^{n-1}}\left(\int_{W(\underline{h})} E\left(\underline{y}-h \underline{m},-h m_{n}\right) e_{n}^{+} g(\underline{y}) d \Gamma_{\underline{y}}-E\left(h\left(\underline{\eta}-\underline{m},-m_{n}\right)\right) e_{n}^{+} g(h \underline{\eta}) h^{n-1}\right)\right| \\
& \quad \leq\left|\sum_{\underline{\eta} \in \mathbb{Z}^{n-1}} \int_{W(h \underline{\eta})} E\left(h\left(\underline{\eta}-\underline{m},-m_{n}\right)\right) e_{n}^{+}[g(h \underline{\eta})-g(\underline{y})] d \Gamma_{\underline{y}}\right| \\
& \quad+\left|\sum_{\underline{\eta} \in \mathbb{Z}^{n-1}} \int_{W(h \underline{\eta})}\left[E\left(h\left(\underline{\eta}-\underline{m},-m_{n}\right)\right)-E\left(\underline{y}-h \underline{m},-h m_{n}\right)\right] e_{n}^{+} g(\underline{y}) d \Gamma_{\underline{y}}\right| .
\end{aligned}
$$

For the estimation of the first term we use the fact that

$$
|E(x)| \leq \frac{\tilde{C}}{|x|^{n-1}}, \quad x \neq 0
$$

together with $1 / p+1 / q=1$. Then, we get

$$
\begin{aligned}
& \left|\sum_{\underline{\eta} \in \mathbb{Z}^{n-1}} \int_{W(\underline{\eta} h)} E\left(h\left(\underline{\eta}-\underline{m},-m_{n}\right)\right) e_{n}^{+}[g(h \underline{\eta})-g(\underline{y})] d \Gamma_{\underline{y}}\right| \\
& \quad \leq c_{1}^{2} \sum_{\underline{\eta} \in \mathbb{Z}^{n-1}}\left(\int_{W(\underline{\eta} h)}\left|E\left(h\left(\underline{\eta}-\underline{m},-m_{n}\right)\right)\right|^{\tilde{q}} d \Gamma_{\bar{y}}\right)^{1 / \tilde{q}}\left(\int_{W(\underline{\eta} h)}|g(h \underline{\eta})-g(\underline{y})|^{\tilde{p}} d \Gamma_{\underline{y}}\right)^{1 / \tilde{p}} \\
& \quad \leq c_{1}^{2}\left(\sum_{\underline{\eta} \in \mathbb{Z}^{n-1}}\left|E\left(h\left(\underline{n}-\underline{m}, m_{n}\right)\right)\right|^{q} h^{(n-1) q / \tilde{q}}\right)^{1 / q}\left(\sum_{\underline{\eta} \in \mathbb{Z}^{n-1}}\left(\int_{w(\underline{n} h)}|g(h \underline{n})-g(\underline{y})|^{\tilde{p}} d \underline{\underline{y}}\right)^{p / \tilde{p}}\right)^{1 / p} \\
& \quad \leq c_{2}\left(\sum_{\underline{\eta} \in \mathbb{Z}^{n-1}}\left(\int_{w(\underline{\eta} h)} \sum_{j=1}^{n} \int_{0}^{1}\left|\partial_{j g}(y+\theta(y-h \underline{\eta}))\right|^{p} d \theta h^{p} d \Gamma_{\underline{y}}\right)\right)^{1 / p}
\end{aligned}
$$

by taking $\tilde{p}=p$, and $\tilde{q}=q>1$ because of $E\left(\cdot-\underline{m},-m_{n}\right) \in l_{q}\left(h \mathbb{Z}^{n-1}\right)$ for any $m=\left(\underline{m}, m_{n}\right)$. Since $g \in W_{p}^{1}\left(\mathbb{R}^{n-1}\right)$ we get

$$
\left|\sum_{\underline{\eta} \in \mathbb{Z}^{n-1}} \int_{W(\underline{\eta} h)} E\left(h\left(\underline{\eta}-\underline{m},-m_{n}\right)\right) e_{n}^{+}[g(h \underline{\eta})-g(\underline{y})] d \Gamma_{\underline{y}}\right| \leq C_{3} h^{1}\|g\|_{W_{p}^{1}},
$$

where all constants are independent of $h$ and $g$. For the second term we apply Hölder's 
inequality $(1 / p+1 / q=1)$ two times and use a Taylor expansion for the kernel. Indeed,

$$
\begin{aligned}
& \left|\sum_{\underline{\eta} \in \mathbb{Z}^{n-1}} \int_{W(h \underline{\eta})}\left[E\left(h\left(\underline{\eta}-\underline{m},-m_{n}\right)\right)-E\left(\underline{y}-h \underline{m},-h m_{n}\right)\right] e_{n}^{+} g(\underline{y}) d \Gamma_{\underline{y}}\right| \\
& \leq c_{1}^{2} \sum_{\underline{\eta} \in \mathbb{Z}^{n-1}}\left(\int_{W(h \underline{\eta})}\left|E\left(h\left(\underline{\eta}-\underline{m},-m_{n}\right)\right)-E\left(\underline{y}-h \underline{m},-h m_{n}\right) \| g(\underline{y})\right| d \Gamma_{\underline{y}}\right) \\
& \leq c_{1}^{2} \sum_{\underline{\eta} \in \mathbb{Z}^{n-1}}\left(\int_{W(h \underline{\eta})}\left|E\left(h\left(\underline{\eta}-\underline{m},-m_{n}\right)\right)-E\left(\underline{y}-h \underline{m},-h m_{n}\right)\right|^{q} d \Gamma_{\underline{y}}\right)^{1 / q}\left(\left.\int_{W(h \bar{\eta})} g(\underline{y})\right|^{p} d \Gamma_{\underline{y}}\right)^{1 / p} \\
& \leq C_{4} \sum_{\underline{\eta} \in \mathbb{Z}^{n-1}}\left(\left.\left.\int_{W(h \underline{\eta})}\left|\sum_{k=1}^{n-1}\right| \partial_{y_{k}} E(\underline{y}-h m)\right|_{y=h \underline{\eta}}\left|y_{k}-h \eta_{k}\right|\right|^{q} d \Gamma_{\underline{y}}\right)^{1 / q}\left(\int_{W(h \underline{\eta})}|g(\underline{y})|^{p} d \Gamma_{\underline{y}}\right)^{1 / p} \\
& \leq C_{4}\left(\left.\left.\sum_{\underline{\eta} \in \mathbb{Z}^{n-1}} \int_{W(h \underline{\eta})}\left|\sum_{k=1}^{n-1}\right| \partial_{y_{k}} E(\underline{y}-h m)\right|_{y=h \underline{\eta}}\left|y_{k}-h \eta_{k}\right|\right|^{q} d \Gamma_{\underline{y}}\right)^{1 / q}\left(\sum_{\underline{\eta} \in \mathbb{Z}^{n-1}} \int_{W(h \underline{\eta})}|g(\underline{y})|^{p} d \Gamma_{\underline{y}}\right)^{1 / p} \\
& \leq \frac{h C_{4}}{2}\left(\left.\left.\sum_{\underline{\eta} \in \mathbb{Z}^{n-1}} \int_{W(h \underline{\eta})}\left|\sum_{k=1}^{n-1}\right| \partial_{y_{k}} E(\underline{y}-h m)\right|_{y=h \underline{\eta}}\right|^{q} d \Gamma_{\underline{y}}\right)^{1 / q}\|g\|_{L_{p}\left(\mathbb{R}^{n-1}\right)}
\end{aligned}
$$

with $C_{4}>0$ a constant independent on $h$ and $g$. Now, we have

$$
E(\underline{y}-h m)=\sum_{j=1}^{n-1} \frac{\overline{y_{j}-h m_{j}}}{|\underline{y}-h m|^{n}} e_{j}+\frac{\overline{-h m_{n}}}{|\underline{y}-h m|^{n}} e_{n}=-\left(\sum_{j=1}^{n-1} \frac{y_{j}-h m_{j}}{|\underline{y}-h m|^{n}} e_{j}-\frac{h m_{n}}{|\underline{y}-h m|^{n}} e_{n}\right)
$$

so that

$$
\begin{gathered}
\sum_{k=1}^{n-1}\left|\partial_{y_{k}} E(\underline{y}-h m)\right|_{y=h \underline{\eta}}=\sum_{k=1}^{n-1}\left|\frac{1}{|\underline{y}-h m|^{n}} e_{k}-n \frac{\left(y_{k}-h m_{k}\right)^{2}}{|\underline{y}-h m|^{n+2}} e_{k}+n h m_{n} \frac{y_{k}-h m_{k}}{|\underline{y}-h m|^{n+2}}\right|_{y=h \underline{\eta}} \\
\quad \leq \frac{n-1}{|\underline{y}-h m|^{n}}+n \frac{1}{|\underline{y}-h m|^{n}}+\left.\frac{n(n-1) h\left|m_{n}\right|}{|\underline{y}-h m|^{n+1}}\right|_{y=h \underline{\eta}} \leq \frac{2 n-1}{|h \underline{\eta}-h m|^{n}}+\frac{n(n-1)\left|h m_{n}\right|}{|h \underline{\eta}-h m|^{n+1}} .
\end{gathered}
$$

Substituting in (36) we obtain

$$
\begin{aligned}
& \frac{h C_{4}}{2}\left(\left.\left.\sum_{\underline{\eta} \in \mathbb{Z}^{n-1}} \int_{W(h \underline{\eta})}\left|\sum_{k=1}^{n-1}\right| \partial_{y_{k}} E(\underline{y}-h m)\right|_{y=h \underline{\eta}}\right|^{q} d \Gamma_{\underline{y}}\right)^{1 / q}\|g\|_{L_{p}\left(\mathbb{R}^{n-1}\right)} \\
& \quad \leq C_{5} h^{1}\|g\|_{L_{p}\left(\mathbb{R}^{n-1}\right)},
\end{aligned}
$$


where again $C_{5}>0$ is a constant independent on $h$ and $g$. This completes the proof of the lemma.

Remark 4.2. The error estimate (32) remains true if $\left(-e_{n}^{+}\right)$is replaced by $\left(-e_{n}^{-}\right)$.

Based on the above lemma we get the following estimate:

Lemma 4.3. Let $g \in H_{p}^{+} \cap C^{\alpha}\left(\mathbb{R}^{n-1}, \mathbb{C}_{n}\right)$, with $0<\alpha \leq 1,1<p<n$. Then, we have

$$
\begin{aligned}
& \left|\int_{\mathbb{R}^{n-1}} E(\underline{y}-h m)\left(-e_{n}^{-}\right) g(\underline{y}) d \Gamma_{\underline{y}}-\sum_{\underline{\eta} \in \mathbb{Z}^{n-1}} E_{h}^{-+}\left(h\left(\underline{\eta}-\underline{m}, 1-m_{n}\right)\right)\left(-e_{n}^{-}\right) \mathscr{A}_{+}\left[-e_{n}^{+} g\right](h \underline{\eta}) h^{n-1}\right| \\
& \quad \leq\left(\tilde{A} h^{n-1}+\tilde{B} h\right)\|g\|_{L_{p}},
\end{aligned}
$$

for all $h m \in h \mathbb{Z}_{+}^{n}$, where again $\tilde{A}, \tilde{B}>0$ are constants independent of $h$ and $g$, and $1 / p+1 / q=1$.

Proof. For an arbitrary $m \in \mathbb{Z}_{+}^{n}$ we estimate this difference by

$$
\begin{aligned}
& \left.\mid \int_{\mathbb{R}^{n-1}} E(\underline{y}-h m)\left(-e_{n}^{-}\right) g \underline{y}\right) d \underline{\Gamma_{\underline{y}}}-\sum_{\underline{\eta} \in \mathbb{Z}^{n-1}} E_{h}^{-+}\left(h\left(\underline{\eta}-\underline{m}, 1-m_{n}\right)\right)\left(-e_{n}^{-}\right) \mathscr{A}_{+}\left[-e_{n}^{+} g\right](h \underline{\eta}) h^{n-1} \mid \\
& \quad \leq\left|\sum_{\underline{\eta} \in \mathbb{Z}^{n-1}}\left(\int_{W(h \underline{\eta})} E\left(\underline{y}-h \underline{m},-h m_{n}\right) e_{n}^{-} g(\underline{y}) d \Gamma_{\underline{y}}-E\left(h\left(\underline{\eta}-\underline{m},-m_{n}\right)\right) e_{n}^{-} g(h \underline{\eta}) h^{n-1}\right)\right| \\
& \quad+\sum_{\underline{\eta} \in \mathbb{Z}^{n-1}}\left|\left(E\left(h\left(\underline{\eta}-\underline{m},-m_{n}\right)\right)-E_{h}^{-+}\left(h\left(\underline{\eta}-\underline{m}, 1-m_{n}\right)\right)\right) e_{n}^{-} g(h \underline{\eta})\right| h^{n-1} \\
& \quad+\sum_{\underline{\eta} \in \mathbb{Z}^{n-1}}\left|E_{h}^{-+}\left(h\left(\underline{\eta}-\underline{m}, 1-m_{n}\right)\right) e_{n}^{-}\left(g(\underline{h} \underline{\eta})-\mathscr{A}_{+}\left[-e_{n}^{+} g\right](h \underline{\eta})\right)\right| h^{n-1}
\end{aligned}
$$

where $W(h \underline{\eta})$ is the hypercube on $\mathbb{R}^{n-1}$ centred in $h \underline{\eta}$ and with size-length $h$. Since the first two expressions are estimated as in the previous lemma these estimates will be omitted here.

Before proceeding with the estimate for the last sum, we remark that when restricted to the lattice, $g$ satisfies $g \in h_{+}^{p}$ so it holds the identity $g=H_{+} g$, and

$$
\mathcal{F}_{h} g=\mathcal{F}_{h} H_{+} g=\left[\frac{\underline{\xi}^{D}}{\underline{d}}\left(e_{n}^{+} \frac{h \underline{d}-\sqrt{4+h^{2} \underline{d}^{2}}}{2}+e_{n}^{-} \frac{2}{h \underline{d}-\sqrt{4+h^{2} \underline{d}^{2}}}\right)\right] \mathcal{F}_{h} g .
$$


Hence,

$$
\begin{gathered}
e_{n}^{-}\left(g-\mathscr{A}_{+}\left[-e_{n}^{+} g\right]\right)=e_{n}^{-} \mathcal{F}_{h}^{-1}\left[\frac{\underline{\underline{\xi}}}{\underline{d}} e_{n}^{+} \frac{h \underline{d}-\sqrt{4+h^{2} \underline{d}^{2}}}{2} \mathcal{F}_{h} g-\frac{\xi^{D}}{\underline{d}} \frac{2}{\sqrt{4+h^{2} \underline{d}^{2}}-h \underline{d}} \mathcal{F}_{h}\left[-e_{n}^{+} g\right]\right] \\
=-e_{n}^{-} e_{n}^{+} \mathcal{F}_{h}^{-1}\left[\frac{\underline{\xi}}{\underline{d}}\left(\frac{h \underline{d}-\sqrt{4+h^{2} \underline{d}^{2}}}{2}+\frac{2}{\sqrt{4+h^{2} \underline{d}^{2}}-h \underline{d}}\right) \mathcal{F}_{h} g\right]=-e_{n}^{-} e_{n}^{+} \mathcal{F}_{h}^{-1}\left[\underline{\xi}^{D} \mathcal{F}_{h} g\right] h .
\end{gathered}
$$

Replacing this expression in the last sum of (39), and using the fact that the inverse of the discrete Fourier transform $\mathcal{F}_{h}^{-1}(=\mathcal{F}$, the continuous Fourier transform restricted to functions in the hypercube $\left.[-(\pi / h), \pi / h]^{n-1}\right)$ has finite operator norm, we get

$$
\begin{aligned}
& \sum_{\underline{\eta} \in \mathbb{Z}^{n-1}}\left|E_{h}^{-+}\left(h\left(\underline{\eta}-\underline{m}, 1-m_{n}\right)\right) e_{n}^{-}\left(g(h \underline{\eta})-\mathscr{A}_{+}\left[-e_{n}^{+} g\right](h \underline{\eta})\right)\right| h^{n-1} \\
& \quad \leq 2^{n+1} h \sum_{\underline{\eta} \in \mathbb{Z}^{n-1}}\left|E_{h}^{-+}\left(h\left(\underline{\eta}-\underline{m}, 1-m_{n}\right)\right) \| \mathcal{F}_{h}^{-1}\left[\underline{\xi}^{D} \mathcal{F}_{h} g\right](h \underline{\eta})\right| h^{n-1} \\
& \leq C_{2} h\left\|E_{h}^{-+}\left(h\left(\cdot, 1-m_{n}\right)\right)\right\|_{l s}\left\|\mathcal{F}_{h}^{-1} \underline{\xi}^{D} \mathcal{F}_{h} g\right\|_{\ell_{p+(n-1) / \alpha}} \\
& \quad \leq C_{3} h\left\|E_{h}^{-+}\right\|_{\ell_{s}}\left\|\mathcal{F}_{h} g\right\|_{\ell_{p+(n-1) / \alpha}} \leq C_{4} h\|g\|_{L_{p}},
\end{aligned}
$$

by arguments similar to the ones in (34), with $1 / s+1 / p(n-1 / \alpha)=1$, and where all the constants involved are positive and independent of $h$ and $g$ (recall, $\left.\underline{\xi}^{D}:=\sum_{j=1}^{n-1}\left(1-e^{i h \xi_{j}} / h\right) e_{j}^{+}-\left(1-e^{-i h \xi_{j}} / h\right) e_{j}^{-}\right)$. This completes our proof.

Both Lemmas 4.1 and 4.3 can now be combined in our main result:

THEOREM 4.4. Let $g \in H_{p}^{+} \cap C^{\alpha}\left(\mathbb{R}^{n-1}, \mathbb{C}_{n}\right) \cap W_{p}^{1}\left(\mathbb{R}^{n-1}, \mathbb{C}_{n}\right)$, for $0<\alpha \leq 1,1<p<n$. Then, the following estimate for the point-wise error between the discrete solution $f_{h}$ of (21) and the continuous solution $f$ of (30) holds:

$$
\left|f(h m)-f_{h}(h m)\right|=\left|\mathcal{C}_{+}[g](m h)-C^{+} \mathcal{G}_{+}[g](m h)\right| \leq\left(A_{+} h^{n-1}+B_{+} h\right)\|g\|_{L_{p}},
$$

for all $m \in \mathbb{Z}_{+}^{n}$, with $A_{+}, B_{+}>0$ constants independent of $h$ and $g$.

Remark 4.5. From the previous proofs we can see that if we lower the conditions in the above theorem to $g \in H_{p}^{+} \cap C^{\alpha}\left(\mathbb{R}^{n-1}, \mathbb{C}_{n}\right), \quad(0<\alpha \leq 1,1<p<n)$, we still get convergence but there is no convergence order with respect to the mesh size $h$. The same can be said about subsequent theorems.

A similar statement can be formulated in the case of lower half space. Let $f \in L_{p}\left(\mathbb{R}_{-}^{n}\right)$ be a solution of

$$
\left\{\begin{array}{cc}
D f(x)=0, & x \in \mathbb{R}_{+}^{n}, \\
f_{-}(\underline{x})=g(\underline{x}), & \underline{x} \in \mathbb{R}^{n-1},
\end{array}\right.
$$

with $\mathbb{R}_{-}^{n}=x \in \mathbb{R}^{n}: x_{n}<0$ and $1<p<+\infty$. Again, $f_{-}$denotes the non-tangential limit 
of $f$ when $y \in \mathbb{R}_{-}^{n}$ goes to $\underline{x} \in \mathbb{R}^{n-1}$. If $g \in H_{p}^{-}$, the Hardy space for the lower half plane, then $f$ is given by

$$
f(x)=\mathcal{C}_{-}[f](x)=\int_{\mathbb{R}^{n-1}} E(\underline{y}-x)\left(-e_{n}\right) g(\underline{y}) d \Gamma_{\underline{y}}, x \in \mathbb{R}_{-}^{n} .
$$

Similar to Theorem 4.4 the following result holds.

THEOREM 4.6. Let $g \in H_{p}^{-} \cap C^{\alpha}\left(\mathbb{R}^{n-1}, \mathbb{C}_{n}\right) \cap W_{p}^{1}\left(\mathbb{R}^{n-1}, \mathbb{C}_{n}\right)$, for $0<\alpha \leq 1,1<p<n$. Then, the following estimate for the point-wise error between the discrete solution $f_{h}$ of the corresponding problem in the lower half lattice and the continuous solution $f$ of (42) holds:

$$
\left|f(h m)-f_{h}(h m)\right|=\left|\mathcal{C}_{-}[g](m h)-C^{-} \mathcal{G}_{-}[g](m h)\right| \leq\left(A_{-} h^{n-1}+B_{-} h\right)\|g\|_{L_{p}},
$$

for all $m \in \mathbb{Z}_{-}^{n}$, with $A_{-}, B_{-}>0$ constants independent of $h$ and $g$.

\subsection{Convergence of related jump problems}

Now, we take a closer look at the jump problems. Here, we consider $f \in L_{p}\left(\mathbb{R}_{+}^{n} \cup \mathbb{R}_{-}^{n}\right)$, with $1<p<+\infty$, to be a solution of

$$
\left\{\begin{array}{cc}
D f(x)=0, & x \in \mathbb{R}_{+}^{n} \cup \mathbb{R}_{-}^{n}, \\
e_{n}^{+} f_{+}(\underline{x})-e_{n}^{-} f_{-}(\underline{x})=e_{n} g(\underline{x}), & \underline{x} \in \mathbb{R}^{n-1},
\end{array}\right.
$$

with $f_{+}, f_{-}$denoting the non-tangential limits of $f$ as in the previous section. For $g \in$ $L_{p}\left(\mathbb{R}^{n-1}\right)$ the solution $f$ can be written as

$$
f(x)=\left\{\begin{array}{cc}
\mathcal{C}_{+}\left[g_{+}\right](x), & x_{n}>0 \\
\mathcal{C}_{-}\left[-g_{-}\right](x), & x_{n}<0
\end{array},\right.
$$

where we recall $e_{n} g=e_{n}^{+} g_{-}+e_{n}^{-} g_{+}=e_{n}^{+}\left(g^{1}+e_{n}^{-} g^{3}\right)+e_{n}^{-}\left(g^{1}-g^{4}+e_{n}^{+} g^{2}\right)$.

Applying Theorems 4.4 and 4.6 , we immediately get the convergence results for Problem II.

Theorem 4.7. Given $g \in L_{p}\left(\mathbb{R}^{n-1}, \mathbb{C}_{n}\right) \cap C^{\alpha}\left(\mathbb{R}^{n-1}, \mathbb{C}_{n}\right) \cap W_{p}^{1}\left(\mathbb{R}^{n-1}, \mathbb{C}_{n}\right), 0<\alpha \leq$ $1,1<p<n$, we have the discrete solution $f_{h}$ of Problem II given by

$$
f_{h}(h m)=\left\{\begin{array}{cc}
C^{+} \mathcal{G}_{+}\left[g_{+}\right](h m), & m_{n} \geq+1, \\
C^{-} \mathcal{G}_{-}\left[-g_{-}\right](h m), & m_{n} \leq-1 .
\end{array}\right.
$$

Moreover, the error between the solution $f$ of the continuous problem (45) and the discrete 
solution $f_{h}$ can be estimated pointwise by

$$
\left|f(h m)-f_{h}(h m)\right| \leq\left(A h^{n-1}+B h\right)\|g\|_{L_{p}},
$$

for all $m \in \mathbb{Z}_{+}^{n} \cup \mathbb{Z}_{-}^{n}$, where $A, B>0$ are constants independent of $h$ and $g$.

Finally, let us consider the convergence results for Problem III.

Theorem 4.8. If $g \in L_{p}\left(\mathbb{R}^{n-1}, \mathbb{C}_{n}\right) \cap C^{\alpha}\left(\mathbb{R}^{n-1}, \mathbb{C}_{n}\right) \cap W_{p}^{1}\left(\mathbb{R}^{n-1}, \mathbb{C}_{n}\right), 0<\alpha \leq$ $1,1<p<n$, then we have the following point-wise estimate between the solution $f_{h}$ of Problem III and the solution $f$ of Problem (45)

$$
\left|f(m h)-f_{h}(m h)\right| \leq C\|g\|_{L_{p}} h^{n-1},
$$

where $C>0$ is a constant independent of $h$ and $g$.

Proof. From Theorem 3.10 we know that the solution to Problem III is given by

$$
f_{h}(h m)= \begin{cases}C^{+}\left[e_{n}^{+} e_{n} R_{h} g, e_{n}^{-} \mathscr{A}_{+}^{-1}\left[-e_{n} R_{h} g\right]\right](h m), & m_{n} \geq+1 \\ C^{-}\left[e_{n}^{-} e_{n} R_{h} g, e_{n}^{+} \mathscr{A}_{-}^{-1}\left[e_{n} R_{h} g\right]\right](h m), & m_{n} \leq-1\end{cases}
$$

where the upper and lower Cauchy transforms are given by

$$
\begin{aligned}
& C^{+}\left[e_{n}^{+} e_{n} R_{h} g, e_{n}^{-} \mathscr{A}_{+}^{-1}\left[-e_{n} R_{h} g\right]\right](h m)=-\sum_{\underline{\eta} \in \mathbb{Z}^{n-1}}\left[E_{h}^{-+}\left(h\left(\underline{\eta}-\underline{m},-m_{n}\right)\right) e_{n}^{-} \mathscr{A}_{+}^{-1}\left[-e_{n} g\right]\right](h \underline{\eta}) \\
& \left.+E_{h}^{-+}\left(h\left(\underline{\eta}-\underline{m}, 1-m_{n}\right)\right) e_{n}^{+} e_{n} g(h \underline{\eta})\right] h^{n-1} \\
& C^{-}\left[e_{n}^{-} e_{n} R_{h} g, e_{n}^{+} \mathscr{A}_{-}^{-1}\left[e_{n} R_{h} g\right]\right](h m)=\sum_{\underline{\eta} \in \mathbb{Z}^{n-1}}\left[E_{h}^{-+}\left(h\left(\underline{\eta}-\underline{m},-1-m_{n}\right)\right) e_{n}^{-} e_{n} g(h \underline{\eta})\right. \\
& \left.\quad+E_{h}^{-+}\left(h\left(\underline{\eta}-\underline{m},-m_{n}\right)\right) e_{n}^{+} \mathscr{A}_{-}^{-1}\left[e_{n} g\right](h \underline{\eta})\right] h^{n-1} .
\end{aligned}
$$

and the operators $\mathscr{A}_{ \pm}^{-1}$ have the representation

$$
\mathscr{A}_{+}^{-1}=\mathcal{F}_{h}^{-1}\left(\frac{\underline{\xi}^{D}}{\underline{d}} \frac{2}{\sqrt{4+h^{2} \underline{d}^{2}}+h \underline{d}}\right) \mathcal{F}_{h}, \quad \mathscr{A}_{-}^{-1}=\mathcal{F}_{h}^{-1}\left(\frac{\sqrt{4+h^{2} \underline{d}^{2}}-h \underline{d}}{\sqrt{4+h^{2} \underline{d}^{2}}+h \underline{d}}\right) \mathcal{F}_{h} .
$$

Now, let us start again with $W(y)$ being a square with centre $y$ and edge length $h$. We only present the estimate for the upper Cauchy transform since the estimate for the lower 
Cauchy transform is similar. For the upper Cauchy transform we have

$$
\begin{aligned}
& \mid \int_{\mathbb{R}^{n-1}} E(\underline{y}-m h)\left(-e_{n}\right) g(\underline{y}) d \Gamma_{\underline{y}}-\sum_{\underline{\eta} \in \mathbb{Z}^{n-1}}\left[E_{h}^{-+}\left(h\left(\underline{\eta}-\underline{m},-m_{n}\right)\right) e_{n}^{-} \mathscr{A}_{+}^{-1}\left[-e_{n} g\right]\right](h \underline{\eta}) \\
& \left.\quad+E_{h}^{-+}\left(h\left(\underline{\eta}-\underline{m}, 1-m_{n}\right)\right) e_{n}^{+} e_{n} g(h \underline{\eta})\right] h^{n-1} \mid \\
& \quad \leq\left|\int_{\mathbb{R}^{n-1}} E(\underline{y}-m h)\left(-e_{n}^{-}\right) g(\underline{y}) d \Gamma_{\underline{y}}-\sum_{\underline{\eta} \in \mathbb{Z}^{n-1}} E_{h}^{-+}\left(h\left(\underline{\eta}-\underline{m},-m_{n}\right)\right)\left(-e_{n}^{-}\right) g(h \underline{\eta}, h) h^{n-1}\right| \\
& \quad+\left|\int_{\mathbb{R}^{n-1}} E(\underline{y}-m h)\left(-e_{n}^{+}\right) g(\underline{y}) d \Gamma_{\underline{y}}-\sum_{\underline{\eta} \in \mathbb{Z}^{n-1}} E_{h}^{-+}\left(h\left(\underline{\eta}-\underline{m}, 1-m_{n}\right)\right)\left(-e_{n}^{+}\right) g(h \underline{\eta}) h^{n-1}\right| \\
& \left.\quad+\mid-\sum_{\underline{\eta} \in \mathbb{Z}^{n-1}} E_{h}^{-+}\left(h\left(\underline{\eta}-\underline{m},-m_{n}\right)\right) e_{n}^{-} \mathscr{A}_{+}^{-1}\left(-e_{n} g\right)\right](h \underline{\eta})+\sum_{\underline{\eta} \in \mathbb{Z}^{n-1}} E_{h}^{-+}\left(h\left(\underline{\eta}-\underline{m},-m_{n}\right)\right)\left(-e_{n}^{-}\right) g(h \underline{\eta}, h) h^{n-1} \mid \\
& \quad+\left|\sum_{\underline{\eta} \in \mathbb{Z}^{n-1}} E_{h}^{-+}\left(h\left(\underline{\eta}-\underline{m}, 1-m_{n}\right)\right) e_{n}^{+} e_{n} g(h \underline{\eta}) h^{n-1}+\sum_{\underline{\eta} \in \mathbb{Z}^{n-1}} E_{h}^{-+}\left(h\left(\underline{\eta}-\underline{m}, 1-m_{n}\right)\right)\left(-e_{n}^{+}\right) g(h \underline{\eta}) h^{n-1}\right| .
\end{aligned}
$$

For the first two terms of formula (49), by Lemma 4.1 we have

$$
\begin{aligned}
& \left|\int_{\mathbb{R}^{n-1}} E(\underline{y}-m h)\left(-e_{n}^{-}\right) g(\underline{y}) d \Gamma_{\underline{y}}-\sum_{\underline{\eta} \in \mathbb{Z}^{n-1}} E_{h}^{-+}\left(h\left(\underline{\eta}-\underline{m},-m_{n}\right)\right)\left(-e_{n}^{-}\right) g(h \underline{\eta}, h) h^{n-1}\right| \\
& \quad \leq\left(A h^{n-1}+B h\right)\|g\|_{L_{p}},
\end{aligned}
$$

and

$$
\begin{aligned}
& \left|\int_{\mathbb{R}^{n-1}} E(\underline{y}-m h)\left(-e_{n}^{+}\right) g(\underline{y}) d \Gamma_{\underline{y}}-\sum_{\underline{\eta} \in \mathbb{Z}^{n-1}} E_{h}^{-+}\left(h\left(\underline{\eta}-\underline{m}, 1-m_{n}\right)\right)\left(-e_{n}^{+}\right) g(h \underline{\eta}) h^{n-1}\right| \\
& \quad \leq\left(A h^{n-1}+B h\right)\|g\|_{L_{p}} h^{n-1} .
\end{aligned}
$$

For the third term in formula (49) we can proceed like in the proof of Lemma 4.3. Using 
Hölder's inequality with $1 / p+1 / q=1$ and $q>n / n-1$ (hence, $1<p<n$ ) we obtain

$$
\begin{aligned}
& \left|-\sum_{\underline{\eta} \in \mathbb{Z}^{n-1}} E_{h}^{-+}\left(h\left(\underline{\eta}-\underline{m},-m_{n}\right)\right) e_{n}^{-} \mathscr{A}_{+}^{-1}\left[-e_{n} g\right](h \underline{\eta})+\sum_{\underline{\eta} \in \mathbb{Z}^{n-1}} E_{h}^{-+}\left(h\left(\underline{\eta}-\underline{m},-m_{n}\right)\right)\left(-e_{n}^{-}\right) g(h \underline{\eta}, h) h^{n-1}\right| \\
& \quad=\left|\sum_{\underline{\eta} \in \mathbb{Z}^{n-1}} E_{h}^{-+}\left(h\left(\underline{\eta}-\underline{m},-m_{n}\right)\right)\left(-e_{n}^{-} \mathscr{A}_{+}^{-1}\left[-e_{n} g\right](h \underline{\eta})+\left(-e_{n}^{-}\right) g(h \underline{\eta})\right) h^{n-1}\right| \\
& \left.\quad \leq 2^{n} \| E_{h}^{-+}\left(\cdot,-h m_{n}\right)\right)\left\|_{\ell_{q}}\right\|-e_{n}^{-} \mathscr{A}_{+}^{-1}\left[-e_{n} g\right]+\left(-e_{n}^{-}\right) g \|_{\ell_{p}} .
\end{aligned}
$$

Using $g=H_{+} g$ we get for the second term

$$
\begin{aligned}
& \left\|-e_{n}^{-} \mathscr{A}_{+}^{-1}\left[-e_{n} g\right]+\left(-e_{n}^{-}\right) g\right\|_{\ell_{p}} \\
& =\| e_{n}^{-} e_{n}^{+} \mathcal{F}_{h}^{-1}\left(\left(\frac{\underline{\xi}}{\underline{d}} \frac{2}{\sqrt{4+h^{2} \underline{d}^{2}}+h \underline{d}}\right)-\left(\frac{\underline{\xi}}{\underline{\underline{g}}} \frac{\sqrt{4+h^{2} \underline{d}^{2}}-h \underline{d}}{2}\right) \mathcal{F}_{h} g \|_{=0} .\right.
\end{aligned}
$$

Therefore, this term vanishes.

For the last term in formula (49)

$(I)=\left|\sum_{\underline{\eta} \in \mathbb{Z}^{n-1}} E_{h}^{-+}\left(h\left(\underline{\eta}-\underline{m}, 1-m_{n}\right)\right) e_{n}^{+} e_{n} g(h \underline{\eta}) h^{n-1}+\sum_{\underline{\eta} \in \mathbb{Z}^{n-1}} E_{h}^{-+}\left(h\left(\underline{\eta}-\underline{m}, 1-m_{n}\right)\right)\left(-e_{n}^{+}\right) g(h \underline{\eta}) h^{n-1}\right|$,

we have the estimate

$$
\begin{aligned}
(I) & =\left|\sum_{\underline{\eta} \in \mathbb{Z}^{n-1}} E_{h}^{-+}\left(h\left(\underline{\eta}-\underline{m}, 1-m_{n}\right)\right)\left(e_{n}^{+} e_{n}+\left(-e_{n}^{+}\right)\right) g(h \underline{\eta}) h^{n-1}\right| \\
& \left.\leq 2^{n}\left\|E_{h}^{-+}\left(\cdot, h\left(1-m_{n}\right)\right)\right\|_{\ell_{q}} \|\left(e_{n}^{+} e_{n}^{-} g-e_{n}^{+} g\right)\right) \|_{\ell_{p}} \\
& \leq 2^{n}\left\|E_{h}^{-+}\left(\cdot, h\left(1-m_{n}\right)\right)\right\|_{\ell_{q}}\left\|e_{n}^{+} e_{n}^{-}\left(1-\frac{\underline{\xi}}{\underline{d}} \frac{2}{h \underline{d}-\sqrt{4+h^{2} \underline{d}^{2}}}\right) \mathcal{F}_{h} g\right\|_{\ell_{p}} \\
& \leq 2^{n}\left\|E_{h}^{-+}\left(\cdot, h\left(1-m_{n}\right)\right)\right\|_{\ell_{q}}\left\|e_{n}^{+} e_{n}^{-} 2 Q^{+} g\right\|_{\ell_{p}}=0 .
\end{aligned}
$$

Collecting all the estimates together we get our result.

\section{Conclusion}

In this paper, we introduced the notion of discrete Hilbert problems in higher dimensions as analogues to the continuous Hilbert problems with respect to null-functions of the Dirac operator. Their solutions are constructed using discrete Cauchy transforms and Hardy decompositions. In the end we show that these discrete Hilbert problems converge to the 
corresponding continuous Hilbert problems when the mesh constant goes to zero and we provide estimates for the convergence order.

\section{Funding}

This work was supported by Portuguese funds through the CIDMA - Center for Research and Development in Mathematics and Applications, and the Portuguese Foundation for Science and Technology ("FCT-Fundação para a Ciência e a Tecnologia"), within project UID/ MAT/04106/2013. The third author is the recipient of a Postdoctoral [grant number SFRH/ $\mathrm{BPD} / 74581 / 2010]$.

\section{Disclosure statement}

No potential conflict of interest was reported by the author(s).

\section{Notes \\ 1. Email: pceres@ua.pt \\ 2. Email: ukaehler@ua.pt}

\section{References}

[1] P. Becher, and H. Joos, The Dirac-Kähler equation and fermions on the lattice, Z. Phys. C 15(4) (1982), pp. 343-365.

[2] S. Bernstein, On the left linear Riemann problem in Clifford analysis, Bull. Belg. Math. Soc. Simon Stevin 3 (1996), pp. 557-576.

[3] F. Brackx, H. De Schepper, F. Sommen, and L. Van de Voorde, Discrete Clifford analysis: a germ of function theory, in Hypercomplex Analysis, I. Sabadini, M. Shapiro, and F. Sommen, eds., Birkhäuser, 2009, pp. 37-53.

[4] P. Cerejeiras, N. Faustino, and N. Vieira, Numerical Clifford analysis for nonlinear Schrödinger problem, Numer. Methods Partial Differ. Equ. 24(4) (2008), pp. 1181-1202, 10. 1002/num.20312.

[5] P. Cerejeiras, U. Kähler, and M. Ku, On the Riemann boundary value problem for null solutions to iterated generalized Cauchy-Riemann operator in Clifford analysis, Results Math. 63(3-4) (2013), pp. 1375-1394.

[6] P. Cerejeiras, U. Kähler, M. Ku, and F. Sommen, Discrete Hardy spaces, J. Fourier Anal. Appl. 20(4) (2014), pp. 715-750, 10.1007/s00041-014-9331-8.

[7] D. Chelkak, and S. Smirnov, Universality in the $2 D$ Ising model and conformal invariance of fermionic observables, Invent. Math. 189(3) (2012), pp. 515-580, 10.1007/s00222-011-03712.

[8] H. De Ridder, H. De Schepper, U. Kähler, and F. Sommen, Discrete function theory based on skew Weyl relations, Proc. Am. Math. Soc. 138(9) (2010), pp. 3241-3256, 10.1090/S00029939-2010-10480-X.

[9] N. Faustino, K. Gürlebeck, A. Hommel, and U. Kähler, Difference potentials for the NavierStokes equations in unbounded domains, J. Differ. Equ. Appl. 12(6) (2006), pp. 577-595, 10. 1080/10236190600637965.

[10] N. Faustino, and U. Kähler, Fischer decomposition for difference dirac operators, Adv. Appl. Cliff. Alg. 17(1) (2007), pp. 37-58, 10.1007/s00006-006-0016-5.

[11] N. Faustino, U. Kähler, and F. Sommen, Discrete Dirac operators in Clifford analysis, Adv. Appl. Cliff. Alg. 17(3) (2007), pp. 451-467, 10.1007/s00006-007-0041-z.

[12] F.D. Gakhov, Boundary value problem, Pergamon Press, Oxford, 1996.

[13] K. Gürlebeck, and A. Hommel, On finite difference Dirac operators and their fundamental solutions, Adv. Appl. Cliff. Alg. 11(S2) (2001), pp. 89-106.

[14] K. Gürlebeck, and W. Sprößig, Quaternionic and Clifford calculus for physicists and engineers, Wiley, 1997.

[15] M. Ku, and U. Kähler, Riemann boundary value problems on half space in Clifford analysis, Math Methods Appl Sci 35(18) (2012), pp. 2141-2156, 10.1002/mma.2557. 
[16] M. Mitrea, Clifford wavelets, singular integrals, and Hardy spaces, Springer, 1994.

[17] N.I. Muskhelishvili, Singular integral equations, 3rd Ed., Nauka, Moscow (in Russian), 1968.

[18] Stanislav Smirnov, Discrete complex analysis and probability, Proceedings of the International Congress of Mathematicians Hyderabad, India 2010.

[19] V. Thomée, Discrete interior Schauder estimates for elliptic difference operators, SIAM J. Numer. Anal. 5 (1968), pp. 626-645.

[20] C. Li, A. McIntosh, and T. Qian, Clifford algebras, Fourier transforms, and singular convolution operators on Lipschitz surfaces, Rev. Mat. Iberoamericana 10 (1994), pp. $665-721$.

[21] I.N. Vekua, Systems of singular integral equations and some boundary value problems, Noordhoff, Groningen, 1967. 